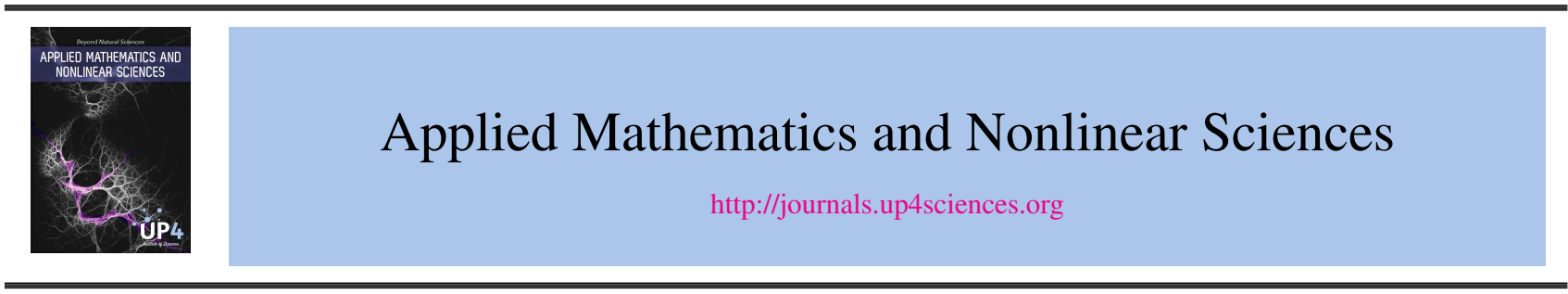

\title{
Global Attractor for Nonlinear Wave Equations with Critical Exponent on Unbounded Domain
}

Yuncheng You ${ }^{\dagger}$

Department of Mathematics and Statistics, University of South Florida, Tampa, FL 33620

USA

Submission Info

Communicated by Tomás Caraballo Received 17th September 2016

Accepted 29th November 2016

Available online 2nd December 2016

\begin{abstract}
Asymptotic and global dynamics of weak solutions for a damped nonlinear wave equation with a critical growth exponent on the unbounded domain $\mathbb{R}^{n}(n \geq 3)$ is investigated. The existence of a global attractor is proved under typical dissipative condition, which features the proof of asymptotic compactness of the solution semiflow in the energy space with critical nonlinear exponent by means of Vitali-type convergence theorem.
\end{abstract}

Keywords: Nonlinear wave equation, global attractor, asymptotic compactness, critical exponent, Vitali-type convergence criterion. AMS 2010 codes: Primary 35B40, 35B41, 35R60, 37L55; Secondary 60H15

\section{Introduction}

In this paper, we study the asymptotic behavior of solutions of a damped semilinear wave equation with nonlinearity of a critical growth exponent over the Euclidean space $\mathbb{R}^{n}$ of arbitrary dimension $n \geq 3$,

$$
u_{t t}-\Delta u+\beta u_{t}+f(x, u)+\alpha u=g(x)
$$

for $t \geq 0$, with the initial condition

$$
u(x, 0)=u_{0}(x), \quad u_{t}(x, 0)=u_{1}(x),
$$

where $\alpha$ and $\beta$ are arbitrary positive constants, $g$ is a given functions defined on $\mathbb{R}^{n}$ and $f(x, u)$ is a nonlinear function satisfying some typical dissipative conditions to be specified.

$\dagger$ Corresponding author: Yuncheng You

Email address: you@mail.usf.edu

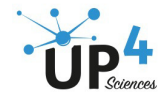


The asymptotic dynamics of global weak solutions for deterministic nonlinear wave equations and for more general nonlinear hyperbolic evolutionary equations with linear or nonlinear damping have been studied in last three decades by many authors, e.g. [1]- [4], [6]- [8], [12]- [14], [16], [19]- [22], [26]. The obtained results focused on the existence of global attractors under certain assumptions.

In the arena of stochastic wave equations driven by additive or multiplicative noise, the solution mapping defines a random dynamical system or called a cocycle on a state space with a parametric base space. The existence of random attractors for stochastic damped wave equations has been studied in [9], [11], [15], [17], [18], [23]- [25].

However, the existence problem of global attractors remains open for damped nonlinear wave equations with nonlinearity of a critical growth exponent and on the unbounded domain $\mathbb{R}^{n}$ with arbitrary dimension. This is the topic of this work.

In case of nonlinearity with higher or critical growth exponents and on the unbounded domain, the issue of asymptotic compactness for the weak or mild solutions of nonlinear damped wave equations becomes difficult to handle due to not only the lack of compactness of the Sobolev embeddings but also the necessarily involved high-order integrable function spaces, in addition to the local existence and regularity of solutions in such spaces. In this work we shall tackle this challenging problem and prove the existence of a global attractor by means of

1) the uniform estimates for absorbing property and norm-smallness of solutions outside a large ball,

2) the esimates of the extended energy functional for the compactness in the space $H^{1}\left(\mathbb{R}^{n}\right) \times L^{2}\left(\mathbb{R}^{n}\right)$ and

3 ) the Vitali-type convergence criterion (Theorem 8) for the function space $L^{p}\left(\mathbb{R}^{n}\right)$ shown in the paper.

This new approach has potential applications to many other nonlinear and stochastic PDEs and to longtime and asymptotic dynamics of various problems with complex and nonlinear interactions.

In Section 2, we briefly recall basic concepts and results related to semiflow and global attractors. In Section 3 , we shall conduct uniform estimates of the weak solutions for absorbing sets and for tail parts. In Section 4, we shall establish the intricate asymptotic compactness of the solution semiflow with respect to the Hilbert energy space $H^{1}\left(\mathbb{R}^{n}\right) \times L^{2}\left(\mathbb{R}^{n}\right)$. In Section 5, we prove the crucial asymptotic compactness of the first component of solutions in $L^{p}\left(\mathbb{R}^{n}\right)$. Then the existence of a global attractor for this nonlinear damped wave equation is finally proved.

In this paper, we shall use $\|\cdot\|$ and $\langle\cdot, \cdot\rangle$ to denote the norm and inner product of $L^{2}\left(\mathbb{R}^{n}\right)$, respectively. The norm of $L^{r}\left(\mathbb{R}^{n}\right)$ with $r \neq 2$ or a Banach space $X$ will be denoted by $\|\cdot\|_{r}$ or $\|\cdot\|_{X}$. We use $c, C$ or $C_{i}$ to denote generic or specific positive constants.

\section{Preliminaries and Assumptions}

Let $\left(X,\|\cdot\|_{X}\right)$ be a real Banach space. The following are the basic concepts and result on the topic of global attractor for infinite dimensional dynamical systems, cf. [2], [8], [20] and [22].

Definition 1. A mapping $\Phi: \mathbb{R}^{+} \times X \rightarrow X$ is called a semiflow on $X$, if the following conditions are satisfied:

(i) $\Phi(0, \cdot)$ is the identity on $X$.

(ii) $\Phi(t+s, \cdot)=\Phi(t, \Phi(s, \cdot))$, for any $t, s \geq 0$.

(iii) $\Phi: \mathbb{R}^{+} \times X \rightarrow X$ is a continuous mapping.

Definition 2. Let $\Phi$ be a semiflow on $X$. A bounded set $K \subset X$ is called an absorbing set for $\Phi$ if for any bounded subset $B \subset X$ there exists a finite time $T_{B}>0$ such that

$$
\Phi(t, B)=\{\Phi(t, x): x \in B\} \subset K, \text { for all } t>T_{B} .
$$

$\Phi$ is called asymptotically compact in $X$ if for any given bounded set $B \subset X$ it holds that

$$
\left\{\Phi\left(t_{m}, x_{m}\right)\right\}_{m=1}^{\infty} \text { has a convergent subsequence in } X,
$$

whenever $t_{m} \rightarrow \infty$ and $\left\{x_{m}\right\}_{m=1}^{\infty} \subset B$. 
Definition 3. Let $\Phi$ be a semiflow on $X$. A set $\mathscr{A} \subset X$ is called a global attractor for $\Phi$, if the following conditions are satisfied:

(i) $\mathscr{A}$ is a compact and invariant set in the sense that $\Phi(t, \mathscr{A})=\mathscr{A}$, for all $t \geq 0$.

(iii) $\mathscr{A}$ attracts every bounded set $B$ in $X$,

$$
\lim _{t \rightarrow \infty} \operatorname{dist}_{X}(\Phi(t, B), \mathscr{A})=0,
$$

where $\operatorname{dist}_{X}(\cdot, \cdot)$ is the Hausdorff semi-distance with respect to the $X$-norm.

Theorem 1. Let $\Phi$ be a semiflow on a Banach space X. If the following two conditions are satisfied:

(1) there is a bounded absorbing set $K$ for the semiflow $\Phi$ in $X$, and

(2) $\Phi$ is asymptotically compact in $X$,

then there exists a global attractor $\mathscr{A}$ in $X$ for the semiflow $\Phi$, which is given by

$$
\mathscr{A}=\omega(K)=\bigcap_{\tau \geq 0} \overline{\bigcup_{t \geq \tau} \Phi(t, K)}
$$

Now we formulate the original initial value problem of the nonlinear damped wave equation (1)-(2). Let $\xi=u_{t}+\delta u$, where $\delta$ is a positive number to be specified later. Then (1)-(2) can be rewritten as

$$
\begin{gathered}
u_{t}+\delta u=v, \\
v_{t}-\delta v+\left(\delta^{2}+\alpha+A\right) u+\beta(v-\delta u)+f(x, u)=g(x) \\
u(x, \tau)=u_{0}(x), v(x, \tau)=v_{0}(x)=u_{1}(x)+\delta u_{0}(x),
\end{gathered}
$$

where the linear operator $A=-\Delta: H^{2}\left(\mathbb{R}^{n}\right) \rightarrow L^{2}\left(\mathbb{R}^{n}\right)$.

Standing Assumption. Throughout the paper, assume that the nonlinear term $f \in C^{1}\left(\mathbb{R}^{n} \times \mathbb{R}, \mathbb{R}\right), n \geq 3$, and its antiderivative $F(x, u)=\int_{0}^{u} f(x, s) d s$ satisfy the following conditions:

$$
\begin{aligned}
& |f(x, u)| \leq C_{1}|u|^{p-1}+\phi_{1}(x), \quad \phi_{1}(x) \in H^{1}\left(\mathbb{R}^{n}\right), \\
& f(x, u) u-C_{2} F(x, u) \geq \phi_{2}(x), \quad \phi_{2}(x) \in L^{1}\left(\mathbb{R}^{n}\right), \\
& F(x, u) \geq C_{3}|u|^{p}-\phi_{3}(x), \quad \phi_{3}(x) \in L^{1}\left(\mathbb{R}^{n}\right),
\end{aligned}
$$

where $C_{1}, C_{2}$ and $C_{3}$ are positive constants and $1 \leq p \leq \frac{n+2}{n-2}$ is arbitrarily given. Assume that $g \in H^{1}\left(\mathbb{R}^{n}\right)$.

Define the phase space

$$
E=\left(H^{1}\left(\mathbb{R}^{n}\right) \cap L^{p}\left(\mathbb{R}^{n}\right)\right) \times L^{2}\left(\mathbb{R}^{n}\right)
$$

endowed with the norm

$$
\|(u, v)\|_{\left(H^{1} \cap L^{p}\right) \times L^{2}}=\left(\|\nabla u\|^{2}+\|u\|^{2}+\|v\|^{2}\right)^{\frac{1}{2}}+\|u\|_{L^{p}}, \quad \text { for }(u, v) \in E .
$$

Lemma 2. For any given $g_{0}=\left(u_{0}, v_{0}\right) \in E$, the initial value problem (4) has a unique global weak solution

$$
\left(u\left(\cdot, u_{0}\right), v\left(\cdot, v_{0}\right)\right) \in C([0, \infty), E) .
$$

Moreover, for any $t \geq 0$, the solution $\left(u\left(t, u_{0}\right), v\left(t, v_{0}\right)\right)$ is weakly continuous with respect to $g_{0}=\left(u_{0}, v_{0}\right) \in E$ in the sense that

$$
\left(u\left(t, u_{0, m}\right), v\left(t, v_{0, m}\right)\right) \rightarrow\left(u\left(t, u_{0}\right), v\left(t, v_{0}\right)\right)
$$

weakly in $E$, provided that $g_{0, m}=\left(u_{0, m}, v_{0, m}\right) \rightarrow g_{0}=\left(u_{0}, v_{0}\right)$ weakly in $E$.

Proof. The local existence and uniqueness of a weak solution for this problem (4) in the phase space $E=$ $\left(H^{1}\left(\mathbb{R}^{n}\right) \cap L^{p}\left(\mathbb{R}^{n}\right)\right) \times L^{2}\left(\mathbb{R}^{n}\right)$ and its weakly continuous dependence on the initial data can be established by the Galerkin approximation method as in [8, Chapter XV] and [3]. Also see [20], [22] and [25]. Here the detail is omitted. The proof of the global existence of weak solutions will be included in the proof of Lemma 3 below. 


\section{Uniform Estimates of Solution Trajectories}

In this section, we shall derive uniform estimates on the solutions of the nonlinear damped wave equation (4) defined on $\mathbb{R}^{n}$ in a long run. These a priori estimates pave the way to proving the existence of absorbing set and the asymptotic compactness of the semiflow $\Phi$. In particular, we will show that tails of the solutions for large spatial variables are uniformly small when time is sufficiently large.

Define a new norm of $E$ by

$$
\|(u, v)\|_{E}=\left(\|v\|^{2}+\left(\alpha+\delta^{2}-\beta \delta\right)\|u\|^{2}+\|\nabla u\|^{2}\right)^{\frac{1}{2}}+\|u\|_{L^{p}},
$$

in which and hereafter let $\delta$ be a fixed positive constant satisfying

$$
\alpha+\delta^{2}-\beta \delta>0 \quad \text { and } \quad \beta-3 \delta>0 .
$$

Obviously the norm $\|\cdot\|_{E}$ in (9) and the Sobolev norm $\|\cdot\|_{\left(H^{1} \cap L^{p}\right) \times L^{2}}$ in (8) are equivalent.

\subsection{Absorbing Set}

The next lemma shows that there exists an absorbing set in the Banach space $E$ for the semiflow $\Phi$ generated by the weak solutions $\left(u\left(t, u_{0}\right),\left(v\left(t, v_{0}\right)\right)\right.$ to the problem $(4)$,

$$
\Phi\left(t, g_{0}\right)=\left(u\left(t, u_{0}\right), v\left(t, v_{0}\right)\right), \quad t \geq 0, g_{0}=\left(u_{0}, v_{0}\right) .
$$

Lemma 3. There exists an absorbing set $K \subset E$ for the solution semiflow $\Phi$ of the problem (4). For any bounded set $B \subset E$, there exists a finite $T_{B}>0$, such that

$$
\Phi(t, B) \subset K, \quad \text { for all } t>T_{B} .
$$

Proof. Take the inner product of the second equation of (4) with $v$ in $L^{2}\left(\mathbb{R}^{n}\right)$ to get

$$
\frac{1}{2} \frac{d}{d t}\|v\|^{2}-\delta\|v\|^{2}+\left(\alpha+\delta^{2}\right)\langle u, v\rangle+\langle A u, v\rangle+\langle f(x, u), v\rangle=-\langle\beta(v-\delta u, v\rangle+\langle g(x), v\rangle .
$$

Then we find that

$$
\langle u, v\rangle=\left\langle u, u_{t}+\delta u\right\rangle=\frac{1}{2} \frac{d}{d t}\|u\|^{2}+\delta\|u\|^{2} \text { and }\langle A u, v\rangle=\frac{1}{2} \frac{d}{d t}\|\nabla u\|^{2}+\delta\|\nabla u\|^{2} .
$$

For the last term on the left-hand side of (11), we have

$$
\langle f(x, u), v\rangle=\frac{d}{d t} \int_{\mathbb{R}^{n}} F(x, u) d x+\delta\langle f(x, u), u\rangle .
$$

By (6), we get

$$
\delta\langle f(x, u), u\rangle \geq \delta C_{2} \int_{\mathbb{R}^{n}} F(x, u) d x+\delta \int_{\mathbb{R}^{n}} \phi_{2} d x .
$$

For the last term on the right-hand side of (11),

$$
\langle g, v\rangle \leq\|g\|\|v\| \leq \frac{\|g\|^{2}}{2(\beta-\delta)}+\frac{\beta-\delta}{2}\|v\|^{2} .
$$

Substitute the above inequalities into (11) to obtain

$$
\begin{aligned}
& \frac{1}{2} \frac{d}{d t}\left[\|v\|^{2}+\left(\alpha+\delta^{2}-\beta \delta\right)\|u\|^{2}+\|\nabla u\|^{2}+2 \int_{\mathbb{R}^{n}} F(x, u) d x\right] \\
& +\frac{\delta}{2}\left[\|v\|^{2}+\left(\alpha+\delta^{2}-\beta \delta\right)\|u\|^{2}+\|\nabla u\|^{2}\right]+\delta C_{2} \int_{\mathbb{R}^{n}} F(x, u) d x \\
\leq & \frac{3 \delta-\beta}{2}\|v\|^{2}+\frac{\|g\|^{2}}{2(\beta-\delta)}+\delta\left\|\phi_{2}\right\|_{L^{1}} \leq \frac{\|g\|^{2}}{2(\beta-\delta)}+\delta\left\|\phi_{2}\right\|_{L^{1}}, \quad t \geq 0 .
\end{aligned}
$$


where the term $(3 \delta-\beta)\|v\|^{2} / 2 \leq 0$ due to (10). Let $\sigma$ be a fixed positive constant:

$$
\sigma=\min \left\{\delta, \delta C_{2}\right\}>0 .
$$

Note that $\int_{\mathbb{R}^{n}}\left(F(x, u)+\phi_{3}(x)\right) d x \geq 0$ due to (7). It follows from (12) and (13) that

$$
\begin{aligned}
& \frac{d}{d t}\left[\|v\|^{2}+\left(\alpha+\delta^{2}-\beta \delta\right)\|u\|^{2}+\|\nabla u\|^{2}+2 \int_{\mathbb{R}^{n}}\left(F(x, u)+\phi_{3}(x)\right) d x\right] \\
& +\sigma\left[\|v\|^{2}+\left(\alpha+\delta^{2}-\beta \delta\right)\|u\|^{2}+\|\nabla u\|^{2}+2 \int_{\mathbb{R}^{n}}\left(F(x, u)+\phi_{3}(x)\right) d x\right] \\
\leq & \frac{\|g\|^{2}}{\beta-\delta}+2 \delta\left(C_{2}\left\|\phi_{3}\right\|_{L^{1}}+\left\|\phi_{2}\right\|_{L^{1}}\right), \quad t \geq 0 .
\end{aligned}
$$

Apply Gronwall inequality to (14) and see that every weak solution of (4) satisfies

$$
\begin{aligned}
& \|v(t)\|^{2}+\left(\alpha+\delta^{2}-\beta \delta\right)\|u(t)\|^{2}+\|\nabla u(t)\|^{2}+2 \int_{\mathbb{R}^{n}}\left(F(x, u(t))+\phi_{3}(x)\right) d x \\
\leq & e^{-\sigma(t-\tau)}\left[\left\|v_{0}\right\|^{2}+\left(\alpha+\delta^{2}-\beta \delta\right)\left\|u_{0}\right\|^{2}+\left\|\nabla u_{0}\right\|^{2}+2 \int_{\mathbb{R}^{n}} F\left(x, u_{0}\right) d x\right] \\
& +2 e^{-\sigma(t-\tau)}\left\|\phi_{3}\right\|_{L^{1}}+\frac{1}{\sigma}\left(2 \delta\left(C_{2}\left\|\phi_{3}\right\|_{L^{1}}+\left\|\phi_{2}\right\|_{L^{1}}\right)+\frac{\|g\|^{2}}{\beta-\delta}\right) .
\end{aligned}
$$

Thus for any given bounded set $B \subset E$ and any $\left(u_{0}, v_{0}\right) \in B$, we have

$$
\begin{aligned}
& \|v(t)\|^{2}+\left(\alpha+\delta^{2}-\beta \delta\right) \| u\left(t\left\|^{2}+\right\| \nabla u(t) \|^{2}+2 \int_{\mathbb{R}^{n}}\left(F(x, u(t))+\phi_{3}(x)\right) d x\right. \\
\leq & e^{-\sigma t}\left[\left\|v_{0}\right\|^{2}+\left(\alpha+\delta^{2}-\beta \delta\right)\left\|u_{0}\right\|^{2}+\left\|\nabla u_{0}\right\|^{2}+2 \int_{\mathbb{R}^{n}} F\left(x, u_{0}\right) d x+2\left\|\phi_{3}\right\|_{L^{1}}\right] \\
& +\frac{1}{\sigma}\left(2 \delta\left(C_{2}\left\|\phi_{3}\right\|_{L^{1}}+\left\|\phi_{2}\right\|_{L^{1}}\right)+\frac{\|g\|^{2}}{\beta-\delta}\right) \quad t \geq 0 .
\end{aligned}
$$

According to the assumption (5) and (6), there exists a constant $c=c\left(C_{1}, C_{2}, \phi_{1}, \phi_{2}\right)>0$ such that

$$
\int_{\mathbb{R}^{n}} F\left(x, u_{0}\right) d x \leq c\left(1+\left\|u_{0}\right\|^{2}+\left\|u_{0}\right\|_{L^{p}}^{p}\right) .
$$

It follows that, for any given bounded set $B \subset E$ and $\left(u_{0}, v_{0}\right) \in B$, there exist a constant $C>0$ and a finite $T_{B}>0$ such that

$$
\begin{aligned}
& e^{-\sigma t} {\left[\left\|v_{0}\right\|^{2}+\left(\alpha+\delta^{2}-\beta \delta\right)\left\|u_{0}\right\|^{2}+\left\|\nabla u_{0}\right\|^{2}+2 \int_{\mathbb{R}^{n}} F\left(x, u_{0}\right) d x+2\left\|\phi_{3}\right\|_{L^{1}}\right] } \\
& \leq C e^{-\sigma t}\left(1+\left\|v_{0}\right\|^{2}+\left\|u_{0}\right\|_{H^{1}}^{2}+\left\|u_{0}\right\|_{L^{p}}^{p}\right) \leq 1, \quad \text { for all } t>T_{B} .
\end{aligned}
$$

Substitute (17) into the right-hand side of the last equality in (16) and note that (7) implies

$$
2 \int_{\mathbb{R}^{n}}\left(F\left(x, u\left(t, u_{0}\right)+\phi_{3}(x)\right) d x \geq 2 C_{3}\left\|u\left(t, u_{0}\right)\right\|_{L^{p}}^{p}\right.
$$

Then it results in

$$
\begin{gathered}
\left\|v\left(t, v_{0}\right)\right\|^{2}+\left(\alpha+\delta^{2}-\beta \delta\right)\left\|u\left(t, u_{0}\right)\right\|^{2}+\left\|\nabla u\left(t, u_{0}\right)\right\|^{2}+2 C_{3}\left\|u\left(t, u_{0}\right)\right\|_{L^{p}}^{p} \\
\leq 1+\frac{1}{\sigma}\left(2 \delta\left(C_{2}\left\|\phi_{3}\right\|_{L^{1}}+\left\|\phi_{2}\right\|_{L^{1}}\right)+\frac{\|g\|^{2}}{\beta-\delta}\right), \quad \text { for } t>T_{B} .
\end{gathered}
$$


The inequality (18) show that $\Phi(t, B) \subset K=B_{E}(0, R)$ for $t>T_{B}$, where the radius of the ball $B_{E}(0, R)$ in $E$ is

$$
\begin{aligned}
R= & \left(\frac{1}{\min \left\{1,\left(\alpha+\delta^{2}-\beta \delta\right)\right\}}\left[1+\frac{1}{\sigma}\left(2 \delta\left(C_{2}\left\|\phi_{3}\right\|_{L^{1}}+\left\|\phi_{2}\right\|_{L^{1}}\right)+\frac{\|g\|^{2}}{\beta-\delta}\right)\right]\right)^{\frac{1}{2}} \\
& +\left(\frac{1}{2 C_{3}}\left[1+\frac{1}{\sigma}\left(2 \delta\left(C_{2}\left\|\phi_{3}\right\|_{L^{1}}+\left\|\phi_{2}\right\|_{L^{1}}\right)+\frac{\|g\|^{2}}{\beta-\delta}\right)\right]\right)^{\frac{1}{p}}
\end{aligned}
$$

Therefore, this set $K=B_{E}(0, R)$ is an absorbing set in the phase space $E$ for the solution semiflow $\Phi$. The proof is completed.

\subsection{Tail Estimates}

Next we conduct uniform estimates on the tail parts of the weak solutions for large spatial and time variables. These estimates play key roles in proving the asymptotic compactness in the space $E$ of the dynamical systems $\Phi$ generated by the nonlinear wave equation (4) on the unbounded domain $\mathbb{R}^{n}$.

Lemma 4. For every bounded set $B \subset E$ and $0<\eta \leq 1$, there exists $T=T(B, \eta)>0$ and $V=V(\eta) \geq 1$ such that the semiflow $\Phi$ generated by the nonlinear damped wave equation (4) satisfies

$$
\|\Phi(t, B)\|_{E\left(\mathbb{R}^{n} \backslash B_{r}\right)}=\max _{g_{0} \in B}\left\|\Phi\left(t, g_{0}\right) \zeta_{B_{r}^{c}}\right\|_{E}<\eta
$$

for all $t>T$ and every $r>V$, where $\zeta_{B_{r}^{c}}(x)$ is the characteristic function of the set $\left\{x \in \mathbb{R}^{n}:|x|>r\right\}$.

Proof. Choose a smooth and nondecreasing function $\rho$ such that $0 \leq \rho(s) \leq 1$ for all $s \in[0, \infty)$ and

$$
\rho(s)= \begin{cases}0, & \text { if } 0 \leq s<1, \\ 1, & \text { if } s>2,\end{cases}
$$

with $0 \leq \rho^{\prime}(s) \leq 2$ for $s \geq 0$. Taking the inner product of the second equation of (4) with $\rho\left(|x|^{2} / r^{2}\right) v$ in $L^{2}\left(\mathbb{R}^{n}\right)$, we get

$$
\begin{aligned}
& \quad \frac{1}{2} \frac{d}{d t} \int_{\mathbb{R}^{n}} \rho\left(\frac{|x|^{2}}{r^{2}}\right)|v|^{2} d x-\delta \int_{\mathbb{R}^{n}} \rho\left(\frac{|x|^{2}}{r^{2}}\right)|v|^{2} d x \\
& \quad+\left(\alpha+\delta^{2}\right) \int_{\mathbb{R}^{n}} \rho\left(\frac{|x|^{2}}{r^{2}}\right) u v d x+\int_{\mathbb{R}^{n}}(A u) \rho\left(\frac{|x|^{2}}{r^{2}}\right) v d x+\int_{\mathbb{R}^{n}} \rho\left(\frac{|x|^{2}}{r^{2}}\right) f(x, u) v d x \\
& =\int_{\mathbb{R}^{n}} \rho\left(\frac{|x|^{2}}{r^{2}}\right) g v d x-\int_{\mathbb{R}^{n}} \rho\left(\frac{|x|^{2}}{r^{2}}\right) \beta(v-\delta u) v d x .
\end{aligned}
$$

Hence we have

$$
\begin{aligned}
& \frac{1}{2} \frac{d}{d t} \int_{\mathbb{R}^{n}} \rho\left(\frac{|x|^{2}}{r^{2}}\right)|v|^{2} d x+\left(\alpha+\delta^{2}-\beta \delta\right) \int_{\mathbb{R}^{n}} \rho\left(\frac{|x|^{2}}{r^{2}}\right) u v d x \\
+ & (\beta-\delta) \int_{\mathbb{R}^{n}} \rho\left(\frac{|x|^{2}}{r^{2}}\right)|v|^{2} d x+\int_{\mathbb{R}^{n}}(A u) \rho\left(\frac{|x|^{2}}{r^{2}}\right) v d x+\int_{\mathbb{R}^{n}} \rho\left(\frac{|x|^{2}}{r^{2}}\right) f(x, u) v d x \\
\leq & \frac{\delta}{2} \int_{\mathbb{R}^{n}} \rho\left(\frac{|x|^{2}}{r^{2}}\right)|v|^{2} d x+\int_{\mathbb{R}^{n}} \rho\left(\frac{|x|^{2}}{r^{2}}\right) g v d x .
\end{aligned}
$$


For the second term on the left-hand side of (23), by (4) we have

$$
\begin{aligned}
& \left(\alpha+\delta^{2}-\beta \delta\right) \int_{\mathbb{R}^{n}} \rho\left(\frac{|x|^{2}}{r^{2}}\right) u v d x=\left(\alpha+\delta^{2}-\beta \delta\right) \int_{\mathbb{R}^{n}} \rho\left(\frac{|x|^{2}}{r^{2}}\right) u\left(u_{t}+\delta u\right) d x \\
\geq & \left(\alpha+\delta^{2}-\beta \delta\right)\left(\frac{1}{2} \frac{d}{d t} \int_{\mathbb{R}^{n}} \rho\left(\frac{|x|^{2}}{r^{2}}\right)|u|^{2} d x+\delta \int_{\mathbb{R}^{n}} \rho\left(\frac{|x|^{2}}{r^{2}}\right)|u|^{2} d x\right) \\
& -\frac{\delta}{2}\left(\alpha+\delta^{2}-\beta \delta\right) \int_{\mathbb{R}^{n}} \rho\left(\frac{|x|^{2}}{r^{2}}\right)|u|^{2} d x .
\end{aligned}
$$

For the fourth term on the left-hand side of (23),

$$
\begin{aligned}
& \int_{\mathbb{R}^{n}}(A u) \rho\left(\frac{|x|^{2}}{r^{2}}\right) v d x=\int_{\mathbb{R}^{n}}(A u) \rho\left(\frac{|x|^{2}}{r^{2}}\right)\left(u_{t}+\delta u\right) d x=\int_{\mathbb{R}^{n}}(\nabla u) \nabla\left(\rho\left(\frac{|x|^{2}}{r^{2}}\right)\left(u_{t}+\delta u\right)\right) d x \\
= & \int_{\mathbb{R}^{n}}(\nabla u) \frac{2 x}{r^{2}} \rho^{\prime}\left(\frac{|x|^{2}}{r^{2}}\right) v d x+\int_{\mathbb{R}^{n}}(\nabla u) \rho\left(\frac{|x|^{2}}{r^{2}}\right) \nabla\left(u_{t}+\delta u\right) d x \\
= & \int_{\mathbb{R}^{n}}(\nabla u) \frac{2 x}{r^{2}} \rho^{\prime}\left(\frac{|x|^{2}}{r^{2}}\right) v d x+\frac{1}{2} \frac{d}{d t} \int_{\mathbb{R}^{n}} \rho\left(\frac{|x|^{2}}{r^{2}}\right)|\nabla u|^{2} d x+\delta \int_{\mathbb{R}^{n}} \rho\left(\frac{|x|^{2}}{r^{2}}\right)|\nabla u|^{2} d x .
\end{aligned}
$$

Since $0 \leq \rho^{\prime}(s) \leq 2$, it follows that

$$
\begin{aligned}
& \int_{\mathbb{R}^{n}}(A u) \rho\left(\frac{|x|^{2}}{r^{2}}\right) v d x \geq-\int_{r \leq|x| \leq \sqrt{2} r} \frac{4|x|}{r^{2}}|(\nabla u) v| d x \\
& +\frac{1}{2} \frac{d}{d t} \int_{\mathbb{R}^{n}} \rho\left(\frac{|x|^{2}}{r^{2}}\right)|\nabla u|^{2} d x+\delta \int_{\mathbb{R}^{n}} \rho\left(\frac{|x|^{2}}{r^{2}}\right)|\nabla u|^{2} d x \\
& \geq-\frac{2 \sqrt{2}}{r} \int_{r \leq|x| \leq \sqrt{2} r}\left(|\nabla u|^{2}+|v|^{2}\right) d x+\frac{1}{2} \frac{d}{d t} \int_{\mathbb{R}^{n}} \rho\left(\frac{|x|^{2}}{r^{2}}\right)|\nabla u|^{2} d x+\frac{\delta}{2} \int_{\mathbb{R}^{n}} \rho\left(\frac{|x|^{2}}{r^{2}}\right)|\nabla u|^{2} d x .
\end{aligned}
$$

For the fifth term on the left-hand side of (23), by (5)-(7), we have

$$
\begin{aligned}
& \int_{\mathbb{R}^{n}} \rho\left(\frac{|x|^{2}}{r^{2}}\right) f(x, u) v d x=\int_{\mathbb{R}^{n}} \rho\left(\frac{|x|^{2}}{r^{2}}\right) f(x, u)\left(u_{t}+\delta u\right) d x \\
\geq & \frac{d}{d t} \int_{\mathbb{R}^{n}} \rho\left(\frac{|x|^{2}}{r^{2}}\right) F(x, u) d x+\delta \int_{\mathbb{R}^{n}} \rho\left(\frac{|x|^{2}}{r^{2}}\right)\left(C_{2} F(x, u)+\phi_{2}(x)\right) d x .
\end{aligned}
$$

For the last term on the right-hand side of (23), we see

$$
\int_{\mathbb{R}^{n}} \rho\left(\frac{|x|^{2}}{r^{2}}\right) g v d x \leq \frac{1}{2(\beta-\delta)} \int_{\mathbb{R}^{n}} \rho\left(\frac{|x|^{2}}{r^{2}}\right)|g|^{2} d x+\frac{\beta-\delta}{2} \int_{\mathbb{R}^{n}} \rho\left(\frac{|x|^{2}}{r^{2}}\right)|v|^{2} d x .
$$

Now substitute (24)-(27) into (23), we obtain

$$
\begin{aligned}
& \frac{1}{2} \frac{d}{d t} \int_{\mathbb{R}^{n}} \rho\left(\frac{|x|^{2}}{r^{2}}\right)\left(|v|^{2}+\left(\alpha+\delta^{2}-\beta \delta\right)|u|^{2}+|\nabla u|^{2}+2 F(x, u)\right) d x \\
& +\frac{\delta}{2} \int_{\mathbb{R}^{n}} \rho\left(\frac{|x|^{2}}{r^{2}}\right)|v|^{2} d x+\frac{\delta}{2} \int_{\mathbb{R}^{n}} \rho\left(\frac{|x|^{2}}{r^{2}}\right)\left(\left(\alpha+\delta^{2}-\beta \delta\right)|u|^{2}+|\nabla u|^{2}\right) d x \\
& +\delta C_{2} \int_{\mathbb{R}^{n}} \rho\left(\frac{|x|^{2}}{r^{2}}\right) F(x, u) d x \\
& \leq \frac{2 \sqrt{2}}{r} \int_{r \leq|x| \leq \sqrt{2} r}\left(|\nabla u|^{2}+|v|^{2}\right) d x+\int_{\mathbb{R}^{n}} \rho\left(\frac{|x|^{2}}{r^{2}}\right)\left(\frac{|g|^{2}}{\beta-\delta}+\delta\left|\phi_{2}\right|\right) d x .
\end{aligned}
$$


Since $g \in L^{2}\left(\mathbb{R}^{n}\right)$ and $\phi_{2}, \phi_{3} \in L^{1}\left(\mathbb{R}^{n}\right)$, for any $\eta>0$, there exists $K_{0}=K_{0}(\eta) \geq 1$ such that for all $r \geq K_{0}$,

$$
\int_{\mathbb{R}^{n}} \rho\left(\frac{|x|^{2}}{r^{2}}\right)\left(\frac{|g|^{2}}{\beta-\delta}+\delta\left|\phi_{2}\right|+2 \sigma\left|\phi_{3}\right|\right) d x \leq \int_{|x| \geq r}\left(\frac{|g|^{2}}{\beta-\delta}+\delta\left|\phi_{2}\right|+2 \sigma\left|\phi_{3}\right|\right) d x<\eta
$$

By (13) and (28)-(29), there exists $K_{1}=K_{1}(\eta) \geq 1$ such that for all $r>K_{1}$,

$$
\begin{aligned}
& \frac{d}{d t} \int_{\mathbb{R}^{n}} \rho\left(\frac{|x|^{2}}{r^{2}}\right)\left(|v|^{2}+\left(\alpha+\delta^{2}-\beta \delta\right)|u|^{2}+|\nabla u|^{2}+2\left(F(x, u)+\phi_{3}\right)\right) d x \\
& +\sigma \int_{\mathbb{R}^{n}} \rho\left(\frac{|x|^{2}}{r^{2}}\right)\left(|v|^{2}+\left(\alpha+\delta^{2}-\beta \delta\right)|u|^{2}+|\nabla u|^{2}+2\left(F(x, u)+\phi_{3}\right)\right) d x \\
\leq & \eta\left[1+\int_{r \leq|x| \leq \sqrt{2} r}\left(|\nabla u|^{2}+|v|^{2}\right) d x\right] .
\end{aligned}
$$

Therefore, for any $t>0$ and $r>K_{1}$, it holds that

$$
\begin{aligned}
& \int_{\mathbb{R}^{n}} \rho\left(\frac{|x|^{2}}{r^{2}}\right)\left[|v(t)|^{2}+\left(\alpha+\delta^{2}-\beta \delta\right)|u(t)|^{2}+|\nabla u(t)|^{2}+2\left(F(x, u(t))+\phi_{3}(x)\right)\right] d x \\
\leq & e^{-\sigma t} \int_{\mathbb{R}^{n}} \rho\left(\frac{|x|^{2}}{r^{2}}\right)\left(\left|v_{0}\right|^{2}+\left(\alpha+\delta^{2}-\beta \delta\right)\left|u_{0}\right|^{2}+\left|\nabla u_{0}\right|^{2}+2\left(F\left(x, u_{0}\right)+\phi_{3}(x)\right)\right) d x \\
& +\frac{\eta}{\sigma}+\eta \int_{0}^{t} e^{-\sigma(t-s)} \int_{r \leq|x| \leq \sqrt{2} r}\left(|\nabla u(s)|^{2}+|v(s)|^{2}\right) d x d s .
\end{aligned}
$$

Next we conduct estimates of the terms on the right-hand side of in (30). For the first term, there exists $T_{1}=T_{1}(B, \eta)>0$ and a constant $C_{4}>0$ such that

$$
\begin{aligned}
& \left.\left.e^{-\sigma t} \int_{\mathbb{R}^{n}} \rho\left(\frac{|x|^{2}}{r^{2}}\right)\left(\left|v_{0}\right|^{2}+\left(\alpha+\delta^{2}-\beta \delta\right) \mid u_{0}\right)\right|^{2}+\left|\nabla u_{0}\right|^{2}+2\left(F\left(x, u_{0}\right)+\phi_{3}\right)\right) d x \\
& \leq e^{-\sigma t} \int_{\mathbb{R}^{n}}\left(\left|v_{0}\right|^{2}+\left(\alpha+\delta^{2}-\beta \delta\right)\left|u_{0}\right|^{2}+\left|\nabla u_{0}\right|^{2}\right) d x \\
& \quad+2 e^{-\sigma t} \int_{\mathbb{R}^{n}}\left[\frac{1}{C_{2}}\left(C_{1}\left|u_{0}\right|^{p}+\left|u_{0}\right|\left|\phi_{1}(x)\right|+\left|\phi_{2}(x)\right|\right)+\left|\phi_{3}(x)\right|\right] d x \\
& \leq C_{4} e^{-\sigma t}\left(\left\|\left(v_{0}, u_{0}\right)\right\|^{2}+\left\|\nabla u_{0}\right\|^{2}+\left\|u_{0}\right\|_{L^{p}}^{p}+\left\|\phi_{1}\right\|^{2}+\left\|\phi_{2}\right\|_{L^{1}}+\left\|\phi_{3}\right\|_{L^{1}}\right)<\eta
\end{aligned}
$$

for all $t>T_{1}$. For the second integral term on the right-hand side of (30), applying the Gronwall inequality to (14) while taking the spatial integral over the region $r \leq|x| \leq \sqrt{2} r$, with (13) in mind, we get

$$
\begin{aligned}
& \int_{-t}^{0} e^{\sigma s} \int_{r \leq|x| \leq \sqrt{2} r}\left(|\nabla u(s)|^{2}+|v(s)|^{2}\right) d x d s \\
\leq & e^{-\sigma(s+t)}\left(\left\|v_{0}\right\|^{2}+\left(\alpha+\delta^{2}-\beta \delta\right)\left\|u_{0}\right\|^{2}+\left\|\nabla u_{0}\right\|^{2}\right) \\
& +2 e^{-\sigma(s+t)} \int_{\mathbb{R}^{n}}\left(F\left(x, u_{0}\right)+\phi_{3}(x)\right) d x+\frac{1}{\sigma}\left(2 \delta\left(C_{2}\left\|\phi_{3}\right\|_{L^{1}}+\left\|\phi_{2}\right\|_{L^{1}}\right)+\frac{1}{\beta-\delta}\|g\|^{2}\right) .
\end{aligned}
$$


Based on (31) and (32), there exists $T_{2}=T_{2}(B, \eta)>0$ such that

$$
\begin{aligned}
& \int_{-t}^{0} e^{\sigma s} \int_{r \leq|x| \leq \sqrt{2} r}\left(|\nabla u(s)|^{2}+|v(s)|^{2}\right) d x d s \\
\leq & C t e^{-\sigma t}\left[\left\|\left(u_{0}, v_{0}\right)\right\|^{2}+\left\|\nabla u_{0}\right\|^{2}+\int_{\mathbb{R}^{n}}\left(F\left(x, u_{0}\right)+\phi_{3}(x)\right) d x\right]+\frac{1}{\sigma}\left(C_{6}+\frac{1}{\beta-\delta}\|g\|^{2}\right) \\
\leq & C t e^{-\sigma t}\left[\left\|\left(u_{0}, v_{0}\right)\right\|^{2}+\left\|\nabla u_{0}\right\|^{2}+\left\|\phi_{3}\right\|_{L^{1}}+\frac{1}{C_{2}}\left(C_{1}\left\|u_{0}\right\|_{L^{p}}^{p}+\left\|u_{0}\right\|^{2}+\left\|\phi_{1}\right\|^{2}+\left\|\phi_{2}\right\|_{L^{1}}\right)\right] \\
& +\frac{1}{\sigma}\left(2 \delta\left(C_{2}\left\|\phi_{3}\right\|_{L^{1}}+\left\|\phi_{2}\right\|_{L^{1}}\right)+\frac{1}{\beta-\delta}\|g\|^{2}\right) \leq M, \quad \text { for all } t \geq T_{2},
\end{aligned}
$$

where the constant

$$
M=1+\frac{1}{\sigma}\left(2 \delta\left(C_{2}\left\|\phi_{3}\right\|_{L^{1}}+\left\|\phi_{2}\right\|_{L^{1}}\right)+\frac{1}{\beta-\delta}\|g\|^{2}\right) .
$$

Now assemble all these estimates and substitute (31) and (33) into (30). It shows that for any bounded set $B$ and any $0<\eta \leq 1$, as long as $r>V=\max \left\{K_{0}, K_{1}\right\}$ and $t>\max \left\{T_{1}, T_{2}\right\}$, one has

$$
\begin{aligned}
& \int_{|x| \geq \sqrt{2} r}\left(|v(t)|^{2}+\left(\alpha+\delta^{2}-\beta \delta\right)|u(t)|^{2}+|\nabla u(t)|^{2}+|u(t)|^{p}\right) d x \\
\leq & \int_{\mathbb{R}^{n}} \rho\left(\frac{|x|^{2}}{r^{2}}\right)\left(|v(t)|^{2}+\left(\alpha+\delta^{2}-\beta \delta\right)|u(t)|^{2}+|\nabla u(t)|^{2}\right) d x \\
& +\frac{2}{C_{3}} \int_{\mathbb{R}^{n}} \rho\left(\frac{|x|^{2}}{r^{2}}\right)\left(F\left(x, u(t)+\phi_{3}(x)\right) d x \leq\left(1+\frac{1}{C_{3}}\right)(2+M) \eta .\right.
\end{aligned}
$$

By (9), the above inequality (34) demonstrates that for any bounded $B \subset E$ it holds that

$$
\begin{aligned}
& \|\Phi(t, B)\|_{E\left(\mathbb{R}^{n} \backslash B_{R}\right)}=\max _{g_{0} \in B}\left\|\Phi\left(t, g_{0}\right)\right\|_{E\left(\mathbb{R}^{n} \backslash B_{R}\right)} \\
\leq & {\left[\left(1+\frac{1}{C_{3}}\right)(2+M) \eta\right]^{1 / 2}+\left[\left(1+\frac{1}{C_{3}}\right)(2+M) \eta\right]^{1 / p}, }
\end{aligned}
$$

where $R=\sqrt{2} r$. (35) implies that (20) is satisfied as stated in this theorem just by renaming $r$ to be $R$ and $\eta$ to be $\left(\left(1+1 / C_{3}\right)(2+M) \eta\right)^{1 / 2}+\left(\left(1+1 / C_{3}\right)(2+M) \eta\right)^{1 / p}$. The proof is completed.

\section{Asymptotic Compactness in $H^{1}\left(\mathbb{R}^{n}\right) \times L^{2}\left(\mathbb{R}^{n}\right)$}

In this section, we shall prove the asymptotic compactness in the space $H^{1}\left(\mathbb{R}^{n}\right) \times L^{2}\left(\mathbb{R}^{n}\right)$ of the solution semiflow $\Phi$ associated with the nonlinear damped wave equation (4).

Lemma 5. The following statements hold for $L^{p}\left(\mathbb{R}^{n}\right)$.

1) For $1 \leq p<\infty$, let $\left\{\psi_{m}\right\}$ be a sequence and $\psi$ be a function in $L^{p}\left(\mathbb{R}^{n}\right)$ such that $\left\|\psi_{m}-\psi\right\|_{L^{p}} \rightarrow 0$ as $m \rightarrow \infty$. Then there exists a subsequence $\left\{\psi_{m_{k}}\right\}$ such that

$$
\lim _{k \rightarrow \infty} \psi_{m_{k}}(x)=\psi(x), \quad \text { a.e. on } \mathbb{R}^{n} .
$$

2) For $1<p<\infty$, if a sequence $\left\{\psi_{m}\right\}$ and a function $\psi$ in $L^{p}\left(\mathbb{R}^{n}\right)$ satisfy the following two conditions:

$$
\lim _{m \rightarrow \infty} \psi_{m}(x)=\psi(x) \text {, a.e. on } \mathbb{R}^{n} \text { and }\left\{\psi_{m}\right\} \text { is bounded in } L^{p}\left(\mathbb{R}^{n}\right),
$$


then $\psi_{m} \rightarrow \psi$ weakly in $L^{p}\left(\mathbb{R}^{n}\right)$, as $m \rightarrow \infty$.

3) For $1<p<\infty$, if a sequence $\left\{\psi_{m}\right\}$ and a function $\psi$ in $L^{p}\left(\mathbb{R}^{n}\right)$ satisfy the following two conditions:

$$
\lim _{m \rightarrow \infty} \psi_{m}(x)=\psi(x) \text {, a.e. on } \mathbb{R}^{n} \text { and } \lim _{m \rightarrow \infty}\left\|\psi_{m}\right\|_{L^{p}}=\|\psi\|_{L^{p}},
$$

then $\lim _{m \rightarrow \infty}\left\|\psi_{m}-\psi\right\|_{L^{p}}=0$.

Proof. Since $\mathbb{R}^{n}$ with the Lebesgue measure is a $\sigma$-finite measure space, the first item is a standard result in Real and Functional Analysis.

For the second item, since $L^{p}\left(\mathbb{R}^{n}\right)$ is a reflexive Banach space for $1<p<\infty$, the boundedness of $\left\{\psi_{m}\right\}$ in $L^{p}\left(\mathbb{R}^{n}\right)$ implies that there is $\varphi \in L^{p}\left(\mathbb{R}^{n}\right)$ such that $\psi_{m} \rightarrow \varphi$ weakly as $m \rightarrow \infty$. By Mazur's lemma, this weak convergence implies there exists a sequence $\left\{\zeta_{m}\right\} \subset L^{p}\left(\mathbb{R}^{n}\right)$ such that

$$
\zeta_{m} \in \operatorname{conv}\left\{\psi_{m}, \psi_{m+1}, \cdots\right\} \text { and } \zeta_{m} \rightarrow \varphi \text { strongly in } L^{p}\left(\mathbb{R}^{n}\right) \text {. }
$$

From the condition $\psi_{m} \rightarrow \psi$ a.e. and $\zeta_{m} \in \operatorname{conv}\left(\bigcup_{i=m}^{\infty} \psi_{i}\right)$, it follows that

$$
\zeta_{m} \rightarrow \psi \text { a.e. in } \mathbb{R}^{n}
$$

On the other hand, by the first statement in this lemma, the strong convergence in (38) implies that there exists a subsequence $\left\{\zeta_{m_{k}}\right\}$ such that $\zeta_{m_{k}} \rightarrow \varphi$ a.e. as $k \rightarrow \infty$. Therefore, (39) leads to $\psi=\varphi$ a.e. on $\mathbb{R}^{n}$ so that $\psi_{m} \rightarrow \psi$ weakly as $m \rightarrow \infty$. The third item is a known result in Functional Analysis, cf. [5, Chapter 4]. Thus the proof is completed.

Let us define the following energy functional on $E$ : for $(u, v) \in E$,

$$
\Gamma(u, v)=\|v\|^{2}+\left(\alpha+\delta^{2}-\beta \delta\right)\|u\|^{2}+\|\nabla u\|^{2}+2 \int_{\mathbb{R}^{n}}\left(F(x, u)+\phi_{3}(x)\right) d x .
$$

Compare (9) and (40), we see that

$$
\Gamma(u, v) \leq\|(u, v)\|_{E}^{2}+2 \int_{\mathbb{R}^{n}}\left(F(x, u)+\phi_{3}(x)\right) d x .
$$

Lemma 6. For every bounded set $B \subset E$ and any integer $k>0$, there exists a constant $M_{1}=M_{1}(B, k)>0$ such that for all $m>M_{1}$ one has $t_{m}>k$ with the property that

$$
\Gamma\left(u\left(t_{m}-t, u_{0, m}\right), v\left(t_{m}-t, v_{0, m}\right)\right) \leq R+1+\frac{1}{\sigma}\left[2 \delta\left(C_{2}\left\|\phi_{3}\right\|_{L^{1}}+\left\|\phi_{2}\right\|_{L^{1}}\right)+\frac{\|g\|^{2}}{\beta-\delta}\right]
$$

for all $t \in[0, k]$ and $\left(u_{0, m}, v_{0, m}\right) \in B$, where the constant $R$ is the same as in (19).

Proof. Integrate the inequality (14) over the time interval $[0, t] \subset[0, k]$, where $\delta \geq \sigma$ by (13). Similar to (18), there exists $M_{1}=M_{1}(B, k)>0$ such that for all $m>M_{1}$ one has $t_{m}>k$ and

$$
\begin{aligned}
& \Gamma\left(u\left(t_{m}-t, u_{0, m}\right), v\left(t_{m}-t, v_{0, m}\right)\right) \leq e^{-\sigma(k-t)} \Gamma\left(u\left(t_{m}-k, u_{0, m}\right), v\left(t_{m}-k, v_{0, m}\right)\right) \\
& +\int_{t}^{k} e^{-\sigma(s-t)}\left(2 \delta\left(C_{2}\left\|\phi_{3}\right\|_{L^{1}}+\left\|\phi_{2}\right\|_{L^{1}}\right)+\frac{\|g\|^{2}}{\beta-\delta}\right) d s \\
\leq & R+1+\frac{1}{\sigma}\left[2 \delta\left(C_{2}\left\|\phi_{3}\right\|_{L^{1}}+\left\|\phi_{2}\right\|_{L^{1}}\right)+\frac{\|g\|^{2}}{\beta-\delta}\right], \quad t \in[0, k] .
\end{aligned}
$$

Therefore, (42) is proved. 
Theorem 7. For every bounded set $B$ and for any sequences $t_{m} \rightarrow \infty$ and $g_{0, m}=\left(u_{0, m}, v_{0, m}\right) \in B$, the sequence $\left\{\Phi\left(t_{m}, g_{0, m}\right)\right\}_{m=1}^{\infty}$ has a strongly convergent subsequence in $H^{1}\left(\mathbb{R}^{n}\right) \times L^{2}\left(\mathbb{R}^{n}\right)$, where $\Phi$ is the solution semiflow generated by the nonlinear damped wave equation (4).

Proof. The proof goes through the following steps.

STEP 1. By Lemma 3, there is a constant $M_{2}=M_{2}(B)>0$ such that for all $m \geq M_{2}$ and $g_{0, m} \in B$, we have

$$
\left\|\Phi\left(t_{m}, g_{0, m}\right)\right\|_{E} \leq R+1
$$

where $R>0$ is given by (19). Then there is $(\tilde{u}, \tilde{v}) \in E$ such that, up to a subsequence and relabeled as the same,

$$
\begin{aligned}
& \Phi\left(t_{m}, g_{0, m}\right) \longrightarrow(\tilde{u}, \tilde{v}) \quad \text { weakly in } E \text { and } \\
& \Phi\left(t_{m}, g_{0, m}\right) \longrightarrow(\tilde{u}, \tilde{v}) \quad \text { weakly in } H^{1}\left(\mathbb{R}^{n}\right) \times L^{2}\left(\mathbb{R}^{n}\right) .
\end{aligned}
$$

Since $E$ is a reflexive and separable Banach space, the weak lower-semicontinuity of the $E$-norm and of the norm of $H^{1}\left(\mathbb{R}^{n}\right) \times L^{2}\left(\mathbb{R}^{n}\right)$ as well implies that

$$
\begin{aligned}
& \liminf _{m \rightarrow \infty}\left\|\Phi\left(t_{m}, g_{0, m}\right)\right\|_{E} \geq\|(\tilde{u}, \tilde{v})\|_{E}, \\
& \liminf _{m \rightarrow \infty}\left\|\Phi\left(t_{m}, g_{0, m}\right)\right\|_{H^{1} \times L^{2}} \geq\|(\tilde{u}, \tilde{v})\|_{H^{1} \times L^{2}} .
\end{aligned}
$$

Next we want to prove that in the Hilbert space $H^{1}\left(\mathbb{R}^{n}\right) \times L^{2}\left(\mathbb{R}^{n}\right)$,

$$
\Phi\left(t_{m}, g_{0, m}\right) \longrightarrow(\tilde{u}, \tilde{v}) \text { strongly. }
$$

It suffices to show that

$$
\limsup _{m \rightarrow \infty}\left\|\Phi\left(t_{m}, g_{0, m}\right)\right\|_{H^{1} \times L^{2}} \leq\|(\tilde{u}, \tilde{v})\|_{H^{1} \times L^{2}} .
$$

If so, then (46) and (48) will lead to $\lim _{m \rightarrow \infty}\left\|\Phi\left(t_{m}, g_{0, m}\right)\right\|_{H^{1} \times L^{2}}=\|(\tilde{u}, \tilde{v})\|_{H^{1} \times L^{2}}$. By the item 3 of Lemma 5, we shall obtain (47).

STEP 2. By Lemma 6 and (7), there exists a constant $C>0$ such that, for any given integer $k>0$ and all $m \geq M_{1}(B, k)$, one has $t_{m}>k$ and

$$
\begin{aligned}
& \left\|\left(u\left(t_{m}-t, u_{0, m}\right), v\left(t_{m}-t, v_{0, m}\right)\right)\right\|_{E} \\
\leq & C\left[R+1+\frac{1}{\sigma}\left(2 \delta\left(C_{2}\left\|\phi_{3}\right\|_{L^{1}}+\left\|\phi_{2}\right\|_{L^{1}}\right)+\frac{\|g\|^{2}}{\beta-\delta}\right)\right]^{1 / 2} \\
+ & C\left[R+1+\frac{1}{\sigma}\left(2 \delta\left(C_{2}\left\|\phi_{3}\right\|_{L^{1}}+\left\|\phi_{2}\right\|_{L^{1}}\right)+\frac{\|g\|^{2}}{\beta-\delta}\right)\right]^{1 / p}, t \in[0, k],
\end{aligned}
$$

for any $\left(u_{0, m}, v_{0, m}\right) \in B$. In particular, (49) is satisfied for $t=k$.

According to Banach-Alaoglu theorem, there exists a sequence $\left\{\tilde{u}_{k}, \tilde{v}_{k}\right\}_{k=1}^{\infty}$ in the space $E$ and subsequences of $\left\{t_{m}\right\}_{m=1}^{\infty}$ and $\left\{\left(u_{0, m}, v_{0, m}\right)\right\}_{m=1}^{\infty}$ again relabeled as the same, such that for every integer $k \geq 1$,

$$
\left(u\left(t_{m}-k, u_{0, m}\right), v\left(t_{m}-k, v_{0, m}\right)\right) \longrightarrow\left(\tilde{u}_{k}, \tilde{v}_{k}\right) \text { weakly in } E,
$$

as $m \rightarrow \infty$, which can be extracted through a diagonal selection procedure as in Real Analysis.

By the weakly continuous dependence on the initial data of the weak solutions stated in Lemma 2, here the weak convergence (50) together with the concatenation

$$
\left(u\left(t_{m}, u_{0, m}\right), v\left(t_{m}, v_{0, m}\right)\right)=\left(u\left(k, u\left(t_{m}-k, u_{0, m}\right)\right), v\left(k, v\left(t_{m}-k, v_{0, m}\right)\right)\right)
$$


implies that for all integers $k \geq 1$, when $m \rightarrow \infty$,

$$
\left(u\left(t_{m}, u_{0, m}\right), v\left(t_{m}, v_{0, m}\right)\right) \longrightarrow\left(u\left(k, \tilde{u}_{k}\right), v\left(k, \tilde{v}_{k}\right)\right) \text { weakly in } E .
$$

Thus (45) and (52) validate the following equality that for all positive integers $k$,

$$
(\tilde{u}, \tilde{v})=\left(u\left(k, \tilde{u}_{k}\right), v\left(k, \tilde{v}_{k}\right)\right) .
$$

By the similar argument from (11) to(12), the weak solutions $(u, v)$ of (4) satisfy

$$
\frac{d}{d t} \Gamma\left(u\left(t, u_{0}\right), v\left(t, v_{0}\right)\right)+2 \sigma \Gamma\left(u\left(t, u_{0}\right), v\left(t, v_{0}\right)\right) \leq G\left(u\left(t, u_{0}\right), v\left(t, v_{0}\right)\right),
$$

where

$$
\begin{gathered}
G(u, v)=-2(\beta-\delta-\sigma)\|v\|^{2}-2(\delta-\sigma)\left(\alpha+\delta^{2}-\beta \delta\right)\|u\|^{2} \\
-2(\delta-\sigma)\|\nabla u\|^{2}+4 \sigma \int_{\mathbb{R}^{n}}\left(F(x, u)+\phi_{3}(x)\right) d x-2 \delta\langle f(x, u), u\rangle+2\langle g, v\rangle .
\end{gathered}
$$

From (53) and (54), for any integer $k \geq 1$ we have

$$
\Gamma(\tilde{u}, \tilde{v}) \leq e^{-2 \sigma k} \Gamma\left(\tilde{u}_{k}, \tilde{v}_{k}\right)+\int_{0}^{k} e^{-2 \sigma \xi} G\left(u\left(\xi, \tilde{u}_{k}\right), v\left(\xi, \tilde{v}_{k}\right)\right) d \xi
$$

STEP 3. On the other hand, from the concatenation (51) and the inequality (54), we obtain

$$
\begin{aligned}
& \Gamma\left(u\left(t_{m}, u_{0, m}\right), v\left(t_{m}, v_{0, m}\right)\right) \leq e^{-2 \sigma k} \Gamma\left(u\left(t_{m}-k, u_{0, m}\right), v\left(t_{m}-k, v_{0, m}\right)\right) \\
& -2(\beta-\delta-\sigma) \int_{0}^{k} e^{-2 \sigma \xi}\left\|v\left(\xi, v\left(t_{m}-k, v_{0, m}\right)\right)\right\|^{2} d \xi \\
& -2(\delta-\sigma)\left(\alpha+\delta^{2}-\beta \delta\right) \int_{0}^{k} e^{-2 \sigma \xi}\left\|u\left(\xi, u\left(t_{m}-k, u_{0, m}\right)\right)\right\|^{2} d \xi \\
& -2(\delta-\sigma) \int_{0}^{k} e^{-2 \sigma \xi}\left\|\nabla u\left(\xi, u\left(t_{m}-k, u_{0, m}\right)\right)\right\|^{2} d \xi \\
& +4 \sigma \int_{0}^{k} e^{-2 \sigma \xi} \int_{\mathbb{R}^{n}}\left(F\left(x, u\left(\xi, u\left(t_{m}-k, u_{0, m}\right)\right)\right)+\phi_{3}(x)\right) d x d \xi \\
& -2 \delta \int_{0}^{k} e^{-2 \sigma \xi} \int_{\mathbb{R}^{n}} f\left(x, u\left(\xi, u\left(t_{m}-k, u_{0, m}\right)\right)\right) u\left(\xi, u\left(t_{m}-k, u_{0, m}\right)\right) d x d \xi \\
& +2 \int_{0}^{k} e^{-2 \sigma \xi} \int_{\mathbb{R}^{n}} g(x) v\left(\xi, v\left(t_{m}-k, v_{0, m}\right)\right) d x d \xi .
\end{aligned}
$$

Below we treat all these terms on the right-hand side of the inequality (57).

1) For the first term on the right-hand side of (57), by (42) in Lemma 6, for all $m \geq M_{1}(B, k)$ we have

$$
\begin{aligned}
& e^{-2 \sigma k} \Gamma\left(u\left(t_{m}-k, u_{0, m}\right), v\left(t_{m}-k, v_{0, m}\right)\right) \\
\leq & e^{-2 \sigma k}\left(R+1+\frac{1}{\sigma} e^{\frac{1}{2} \sigma k}\left[2 \delta\left(C_{2}\left\|\phi_{3}\right\|_{L^{1}}+\left\|\phi_{2}\right\|_{L^{1}}\right)+\frac{\|g\|^{2}}{\beta-\delta}\right]\right) \\
\leq & e^{-\sigma k}\left(R+1+\frac{1}{\sigma}\left[2 \delta\left(C_{2}\left\|\phi_{3}\right\|_{L^{1}}+\left\|\phi_{2}\right\|_{L^{1}}\right)+\frac{\|g\|^{2}}{\beta-\delta}\right]\right) .
\end{aligned}
$$


2) For the second term on the right-hand side of (57), by (50) and the weakly continuous dependence of solutions on the initial data stated in Lemma 2, we find that for any $\xi \in[0, k]$, when $m \rightarrow \infty$,

$$
v\left(\xi, v\left(t_{m}-k, v_{0, m}\right)\right) \longrightarrow v\left(\xi, \tilde{v}_{k}\right) \quad \text { weakly in } L^{2}\left(\mathbb{R}^{n}\right),
$$

which implies that for all $\xi \in[0, k]$,

$$
\liminf _{m \rightarrow \infty}\left\|v\left(\xi, v\left(t_{m}-k, v_{0, m}\right)\right)\right\|^{2} \geq\left\|v\left(\xi, \tilde{v}_{k}\right)\right\|^{2} .
$$

By (59) and Fatou's lemma we obtain

$$
\begin{aligned}
& \liminf _{m \rightarrow \infty} \int_{0}^{k} e^{-2 \sigma \xi}\left\|v\left(\xi, v\left(t_{m}-k, v_{0, m}\right)\right)\right\|^{2} d \xi \\
\geq & \int_{0}^{k} e^{-2 \sigma \xi} \liminf _{m \rightarrow \infty}\left\|v\left(\xi, v\left(t_{m}-k, v_{0, m}\right)\right)\right\|^{2} d \xi \geq \int_{0}^{k} e^{-2 \sigma \xi}\left\|v\left(\xi, \tilde{v}_{k}\right)\right\|^{2} d \xi .
\end{aligned}
$$

Since (10) and (13) implies $\beta-\delta-\sigma \geq \beta-2 \delta>0$, (60) leads to

$$
\begin{aligned}
& \limsup _{m \rightarrow \infty}-2(\beta-\delta-\sigma) \int_{0}^{k} e^{-2 \sigma \xi}\left\|v\left(\xi, v\left(t_{m}-k, v_{0, m}\right)\right)\right\|^{2} d \xi \\
= & -2(\beta-\delta-\sigma) \liminf _{m \rightarrow \infty} \int_{0}^{k} e^{-2 \sigma \xi}\left\|v\left(\xi, v\left(t_{m}-k, v_{0, m}\right)\right)\right\|^{2} d \xi \\
\leq & -2(\beta-\delta-\sigma) \int_{0}^{k} e^{-2 \sigma \xi}\left\|v\left(\xi, \tilde{v}_{k}\right)\right\|^{2} d \xi .
\end{aligned}
$$

Similarly for the third and fourth terms, by (50) and Fatou's lemma we obtain

$$
\begin{aligned}
& \limsup _{m \rightarrow \infty}-2(\delta-\sigma)\left(\alpha+\delta^{2}-\beta \delta\right) \int_{0}^{k} e^{-2 \sigma \xi}\left\|u\left(\xi, u\left(t_{m}-k, u_{0, m}\right)\right)\right\|^{2} d \xi \\
& \leq-2(\delta-\sigma)\left(\alpha+\delta^{2}-\beta \delta\right) \int_{0}^{k} e^{-2 \sigma \xi}\left\|u\left(\xi, \tilde{u}_{k}\right)\right\|^{2} d \xi \\
& \limsup _{m \rightarrow \infty}-2(\delta-\sigma) \int_{0}^{k} e^{-2 \sigma \xi}\left\|\nabla u\left(\xi, u\left(t_{m}-k, u_{0, m}\right)\right)\right\|^{2} d \xi \\
& \leq-2(\delta-\sigma) \int_{0}^{k} e^{-2 \sigma \xi}\left\|\nabla u\left(\xi, \tilde{u}_{k}\right)\right\|^{2} d \xi .
\end{aligned}
$$

3) For the fifth term on the right-hand side of (57), we have

$$
\begin{aligned}
& \left|\int_{0}^{k} e^{-2 \sigma \xi} \int_{\mathbb{R}^{n}}\left(F\left(x, u\left(\xi, u\left(t_{m}-k, u_{0, m}\right)\right)\right)-F\left(x, u\left(\xi, \tilde{u}_{k}\right)\right)\right) d x d \xi\right| \\
\leq & \int_{0}^{k} e^{-2 \sigma \xi} \int_{|x|>r}\left|F\left(x, u\left(\xi, u\left(t_{m}-k, u_{0, m}\right)\right)\right)-F\left(x, u\left(\xi, \tilde{u}_{k}\right)\right)\right| d x d \xi \\
+ & \int_{0}^{k} e^{-2 \sigma \xi} \int_{|x| \leq r}\left|F\left(x, u\left(\xi, u\left(t_{m}-k, u_{0, m}\right)\right)\right)-F\left(x, u\left(\xi, \tilde{u}_{k}\right)\right)\right| d x d \xi .
\end{aligned}
$$

A) For any given $\eta>0$, by the proof of Lemma 4 adapted to the time interval $[k, \infty)$, there exist $M_{3}=$ $M_{3}(B, \eta)>M_{2}$ and $K=K(B, \eta) \geq 1$ such that for $\xi \in[0, k]$, whenever $r>K$ and $m>M_{3}$, one has

$$
\int_{|x|>r}\left(\left|u\left(t_{m}-\xi, u_{0, m}\right)\right|^{2}+\left|u\left(t_{m}-\xi, u_{0, m}\right)\right|^{p}+\left|\phi_{1}\right|^{2}+\left|\phi_{2}\right|+\left|\phi_{3}\right|\right) d x<\eta .
$$


In view of (5) and (6), there is a constant $L_{1}>0$ such that for any $\xi \in[0, k]$ one has

$$
\begin{gathered}
\int_{|x|>r}\left|F\left(x, u\left(t_{m}-\xi, u_{0, m}\right)\right)\right| d x \\
\leq \int_{|x|>r} L_{1}\left(\left|u\left(t_{m}-\xi, u_{0, m}\right)\right|^{2}+\left|u\left(t_{m}-\xi, u_{0, m}\right)\right|^{p}+\left|\phi_{1}\right|^{2}+\left|\phi_{2}\right|+\left|\phi_{3}\right|\right) d x<L_{1} \eta,
\end{gathered}
$$

for all $r>K$ and $m>M_{3}$.

B) Since (50) shows that

$$
\tilde{u}_{k}=(\text { weak }) \lim _{m \rightarrow \infty} u\left(t_{m}-k, u_{0, m}\right) \text { in } L^{2}\left(\mathbb{R}^{n}\right) \cap L^{p}\left(\mathbb{R}^{n}\right),
$$

by the weakly continuous dependence of solutions on intial data stated in Lemma 2, by the weak lowersemicontinuity of the $L^{2}$ and $L^{p}$ norms, it yields from (64) that

$$
\begin{aligned}
& \int_{0}^{k} e^{-2 \sigma \xi} \int_{|x|>r}\left|F\left(x, u\left(k-\xi, \tilde{u}_{k}\right)\right)\right| d x d \xi \\
\leq & \int_{0}^{k} e^{-2 \sigma \xi} \int_{|x|>r} L_{1}\left(\left|u\left(k-\xi, \tilde{u}_{k}\right)\right|^{2}+\left|u\left(k-\xi, \tilde{u}_{k}\right)\right|^{p}+\left|\phi_{1}\right|^{2}+\left|\phi_{2}\right|+\left|\phi_{3}\right|\right) d x d \xi \\
= & \int_{0}^{k} e^{-2 \sigma \xi} L_{1}\left(\left\|u\left(k-\xi, \tilde{u}_{k}\right)\right\|_{L^{2}\left(\mathbb{R}^{n} \backslash B_{r}\right)}^{2}+\left\|u\left(k-\xi, \tilde{u}_{k}\right)\right\|_{L^{p}\left(\mathbb{R}^{n} \backslash B_{r}\right)}^{p}\right) d \xi \\
& +\int_{0}^{k} e^{-2 \sigma \xi} L_{1} \int_{|x|>r}\left(\left|\phi_{1}\right|^{2}+\left|\phi_{2}\right|+\left|\phi_{3}\right|\right) d x d \xi \\
\leq & \int_{0}^{k} e^{-2 \sigma \xi} L_{1}\left[\liminf _{m \rightarrow \infty}\left\|u\left(k-\xi, \tilde{u}_{k}\right)\right\|_{L^{2}\left(\mathbb{R}^{n} \backslash B_{r}\right)}+\liminf _{m \rightarrow \infty}\left\|u\left(k-\xi, \tilde{u}_{k}\right)\right\|_{L^{p}\left(\mathbb{R}^{n} \backslash B_{r}\right)}^{p}\right] d \xi \\
& +\int_{0}^{k} e^{-2 \sigma \xi} L_{1} \int_{|x|>r}\left(\left|\phi_{1}\right|^{2}+\left|\phi_{2}\right|+\left|\phi_{3}\right|\right) d x d \xi \leq \frac{L_{1}}{2 \sigma} \eta, \quad \text { for } r>K, m>M_{3} .
\end{aligned}
$$

The above two inequalities show that there exists a constant $L_{2}=L_{1}(1+1 /(2 \sigma))>0$ such that the first term on the right-hand side of (63) satisfies

$$
\begin{aligned}
& \int_{0}^{k} e^{-2 \sigma \xi} \int_{|x|>r}\left|F\left(x, u\left(k-\xi, u\left(t_{m}-k, u_{0, m}\right)\right)\right)-F\left(x, u\left(k-\xi, \tilde{u}_{k}\right)\right)\right| d x d \xi \\
\leq & \int_{0}^{k} e^{-2 \sigma \xi} \int_{|x|>r}\left(\left|F\left(x, u\left(t_{m}-\xi, u_{0, m}\right)\right)\right|+\left|F\left(x, u\left(k-\xi, \tilde{u}_{k}\right)\right)\right|\right) d x d \xi \leq L_{2} \eta
\end{aligned}
$$

for all $r>K$ and $m>M_{3}$.

C) For the second term on the right-hand side of (63), by (50) we have

$$
u\left(k-\xi, u\left(t_{m}-k, u_{0, m}\right)\right) \longrightarrow u\left(k-\xi, \tilde{u}_{k}\right) \text { weakly in } H^{1}\left(\mathbb{B}_{r}\right) \cap L^{p}\left(\mathbb{B}_{r}\right) .
$$

Since $H^{1}\left(\mathbb{B}_{r}\right)$ is compactly embedded in $L^{2}\left(\mathbb{B}_{r}\right)$, it follows that for any $\xi \in[0, k]$,

$$
u\left(k-\xi, u\left(t_{m}-k, u_{0, m}\right)\right) \longrightarrow u\left(k-\xi, \tilde{u}_{k}\right) \text { strongly in } L^{2}\left(\mathbb{B}_{r}\right) .
$$

Then by the first item of Lemma 5 and the continuity of $F(x, u)$,

$$
F\left(x, u\left(k-\xi, u\left(t_{m}-k, u_{0, m}\right)\right)\right) \longrightarrow F\left(x, u\left(k-\xi, \tilde{u}_{k}\right)\right) \text { in } \mathbb{B}_{r}, \text { as } m \rightarrow \infty .
$$


On the other hand, by the Standing Assumption and Lemma 6, we have the following uniform bound that there is a constant $L_{3}>0$ such that

$$
\begin{aligned}
& \int_{|x|<r}\left|F\left(x, u\left(k-\xi, u\left(t_{m}-k, u_{0, m}\right)\right)\right)\right| d x \leq L_{1}\left(\left\|u\left(k-\xi, u\left(t_{m}-k, u_{0, m}\right)\right)\right\|_{L^{2}\left(B_{r}\right)}^{2}\right. \\
& \left.\quad+\left\|u\left(k-\xi, u\left(t_{m}-k, u_{0, m}\right)\right)\right\|_{L^{p}\left(B_{r}\right)}^{p}+\left\|\phi_{1}\right\|^{2}+\left\|\phi_{2}\right\|_{L^{1}\left(\mathbb{R}^{n}\right)}+\left\|\phi_{3}\right\|_{L^{1}\left(\mathbb{R}^{n}\right)}\right) \\
& \leq L_{3}\left[R+1+\frac{1}{\sigma} e^{\frac{1}{2} \sigma k}\left(2 \delta\left(C_{2}\left\|\phi_{3}\right\|_{L^{1}}+\left\|\phi_{2}\right\|_{L^{1}}\right)+\frac{\|g\|^{2}}{\beta-\delta}\right)+\left\|\phi_{1}\right\|^{2}+\left\|\phi_{2}\right\|_{L^{1}}+\left\|\phi_{3}\right\|_{L^{1}}\right]
\end{aligned}
$$

for any $\xi \in[0, k]$ and $m>M_{1}$. By the second item of Lemma 6, it follows from (67) and (68) that

$$
F\left(x, u\left(k-\xi, u\left(t_{m}-k, u_{0, m}\right)\right)\right) \longrightarrow F\left(x, u\left(k-\xi, \tilde{u}_{k}\right)\right) \text { weakly in } L^{1}\left(\mathbb{B}_{r}\right),
$$

as $m \rightarrow \infty$. Consequently, when $m \rightarrow \infty$,

$$
\int_{|x|<r} F\left(x, u\left(k-\xi, u\left(t_{m}-k, u_{0, m}\right)\right)\right) d x \longrightarrow \int_{|x|<r} F\left(x, u\left(k-\xi, \tilde{u}_{k}\right)\right) d x .
$$

Furthermore, (68) shows that

$$
\begin{aligned}
& \left|\int_{|x|<r}\left[F\left(x, u\left(k-\xi, u\left(t_{m}-k, u_{0, m}\right)\right)\right)-F\left(x, u\left(k-\xi, \tilde{u}_{k}\right)\right)\right] d x\right| \\
\leq & L_{3}\left[R+1+\frac{1}{\sigma} e^{\frac{1}{2} \sigma k}\left(2 \delta\left(C_{2}\left\|\phi_{3}\right\|_{L^{1}}+\left\|\phi_{2}\right\|_{L^{1}}\right)+\frac{\|g\|^{2}}{\beta-\delta}\right)+\left\|\phi_{1}\right\|^{2}+\left\|\phi_{2}\right\|_{L^{1}}+\left\|\phi_{3}\right\|_{L^{1}}\right] \\
& +\left\|F\left(\cdot, u\left(k-\xi, \tilde{u}_{k}\right)\right)\right\|_{L^{1}\left(\mathbb{R}^{n}\right)} .
\end{aligned}
$$

According to Lebesgue dominated convergence theorem, (69) and (70) imply that for every integer $k \geq 1$ and any $r \geq K$,

$$
\begin{gathered}
\lim _{m \rightarrow \infty} \int_{0}^{k} e^{-2 \sigma \xi} \int_{|x|<r} F\left(x, u\left(k-\xi, u\left(t_{m}-k, u_{0, m}\right)\right)\right) d x d \xi \\
=\int_{0}^{k} e^{-2 \sigma \xi} \int_{|x|<r} F\left(x, u\left(k-\xi, \tilde{u}_{k}\right)\right) d x d \xi .
\end{gathered}
$$

Put together (63), (65) and (71). Then we obtain

$$
\begin{gathered}
\lim _{m \rightarrow \infty} \int_{0}^{k} e^{-2 \sigma \xi} \int_{\mathbb{R}^{n}}\left(F\left(x, u\left(k-\xi, u\left(t_{m}-k, u_{0, m}\right)\right)\right)+\phi_{3}(x)\right) d x d \xi \\
=\int_{0}^{k} e^{-2 \sigma \xi} \int_{\mathbb{R}^{n}}\left(F\left(x, u\left(k-\xi, \tilde{u}_{k}\right)\right)+\phi_{3}(x)\right) d x d \xi .
\end{gathered}
$$

4) By an argument similar to the proof of (72), we can also prove the convergence of the sixth term on the right-hand side of (57),

$$
\begin{gathered}
\lim _{m \rightarrow \infty} \int_{0}^{k} e^{-2 \sigma \xi} \int_{\mathbb{R}^{n}} f\left(x, u\left(k-\xi, u\left(t_{m}-k, u_{0, m}\right)\right)\right) u\left(k-\xi, u\left(t_{m}-k, u_{0, m}\right)\right) d x d \xi \\
=\int_{0}^{k} e^{-2 \sigma \xi} \int_{\mathbb{R}^{n}} f\left(x, u\left(k-\xi, \tilde{u}_{k}\right)\right) u\left(k-\xi, \tilde{u}_{k}\right) d x d \xi \\
\lim _{m \rightarrow \infty} \int_{0}^{k} e^{-2 \sigma \xi} \int_{\mathbb{R}^{n}} g(x) v\left(k-\xi, v\left(t_{m}-k, v_{0, m}\right)\right) d x d \xi=\int_{0}^{k} e^{-2 \sigma \xi} \int_{\mathbb{R}^{n}} g(x) v\left(k-\xi, \tilde{v}_{k}\right) d x d \xi .
\end{gathered}
$$


STEP 4. Take the limit of (57) as $m \rightarrow \infty$ and assemble together the results shown above in the items 1) through 5) of Step 3. Then we get

$$
\begin{aligned}
& \limsup _{m \rightarrow \infty} \Gamma\left(u\left(t_{m}, u_{0, m}\right), v\left(t_{m}, v_{0, m}\right)\right) \leq e^{-\sigma k}\left(R+1+\frac{1}{\sigma}\left[2 \delta\left(C_{2}\left\|\phi_{3}\right\|_{L^{1}}+\left\|\phi_{2}\right\|_{L^{1}}\right)+\frac{\|g\|^{2}}{\beta-\delta}\right]\right) \\
& -2(\beta-\delta-\sigma) \int_{0}^{k} e^{-2 \sigma \xi}\left\|v\left(k-\xi, \tilde{v}_{k}\right)\right\|^{2} d \xi-2(\delta-\sigma)\left(\alpha+\delta^{2}-\beta \delta\right) \int_{0}^{k} e^{-2 \sigma \xi}\left\|u\left(k-\xi, \tilde{u}_{k}\right)\right\|^{2} d \xi \\
& -2(\delta-\sigma) \int_{0}^{k} e^{-2 \sigma \xi}\left\|\nabla u\left(k-\xi, \tilde{u}_{k}\right)\right\|^{2} d \xi+4 \sigma \int_{0}^{k} e^{-2 \sigma \xi} \int_{\mathbb{R}^{n}}\left(F\left(x, u\left(k-\xi, \tilde{u}_{k}\right)\right)+\phi_{3}(x)\right) d x d \xi \\
& +2 \int_{0}^{k} e^{-2 \sigma \xi} \int_{\mathbb{R}^{n}}\left[g(x) v\left(k-\xi, \tilde{v}_{k}\right)-\delta f\left(x, u\left(k-\xi, \tilde{u}_{k}\right)\right) u\left(k-\xi, \tilde{u}_{k}\right)\right] d x d \xi .
\end{aligned}
$$

From (56) and (73) it follows that

$$
\begin{aligned}
& \limsup _{m \rightarrow \infty} \Gamma\left(u\left(t_{m}, u_{0, m}\right), v\left(t_{m}, v_{0, m}\right)\right) \\
\leq & e^{-\sigma k}\left(R+1+\frac{1}{\sigma}\left[2 \delta\left(C_{2}\left\|\phi_{3}\right\|_{L^{1}}+\left\|\phi_{2}\right\|_{L^{1}}\right)+\frac{\|g\|^{2}}{\beta-\delta}\right]\right)+\int_{0}^{k} e^{-2 \sigma \xi} G\left(u\left(k-\xi, \tilde{u}_{k}\right), v\left(k-\xi, \tilde{v}_{k}\right)\right) d \xi \\
= & e^{-\sigma k}\left(R+1+\frac{1}{\sigma}\left[2 \delta\left(C_{2}\left\|\phi_{3}\right\|_{L^{1}}+\left\|\phi_{2}\right\|_{L^{1}}\right)+\frac{\|g\|^{2}}{\beta-\delta}\right]\right)+\Gamma(\tilde{u}, \tilde{v})-e^{-2 \sigma k} \Gamma\left(\tilde{u}_{k}, \tilde{v}_{k}\right) \\
\leq & e^{-\sigma k}\left(R+1+\frac{1}{\sigma}\left[2 \delta\left(C_{2}\left\|\phi_{3}\right\|_{L^{1}}+\left\|\phi_{2}\right\|_{L^{1}}\right)+\frac{\|g\|^{2}}{\beta-\delta}\right]\right)+\Gamma(\tilde{u}, \tilde{v}) .
\end{aligned}
$$

Take limit $k \rightarrow \infty$ of the above inequality to obtain

$$
\limsup _{m \rightarrow \infty} \Gamma\left(u\left(t_{m}, u_{0, m}\right), v\left(t_{m}, v_{0, m}\right)\right) \leq \Gamma(\tilde{u}, \tilde{v}) .
$$

On the other hand, from (53), (67) and (68) we have

$$
\lim _{m \rightarrow \infty} \int_{\mathbb{R}^{n}} F\left(x, u\left(t_{m}, u_{0, m}\right)\right) d x=\int_{\mathbb{R}^{n}} F(x, \tilde{u}) d x,
$$

which along with (74) shows that

$$
\begin{aligned}
& \limsup _{m \rightarrow \infty}\left(\left\|v\left(t_{m}, v_{0, m}\right)\right\|^{2}+\left(\alpha+\delta^{2}-\beta \delta\right)\left\|u\left(t_{m}, u_{0, m}\right)\right\|^{2}+\left\|\nabla u\left(t_{m}, u_{0, m}\right)\right\|^{2}\right) \\
& \leq\|\tilde{v}\|^{2}+\left(\alpha+\delta^{2}-\beta \delta\right)\|\tilde{u}\|^{2}+\|\nabla \tilde{u}\|^{2} .
\end{aligned}
$$

STEP 5. Note that the norm of $H^{1}\left(\mathbb{R}^{n}\right) \times L^{2}\left(\mathbb{R}^{n}\right)$ is equivalent to

$$
\|(u, v)\|_{\Pi} \stackrel{\text { def }}{=} \Gamma(u, v)-2 \int_{\mathbb{R}^{n}}\left(F(x, u)+\phi_{3}(x)\right) d x=\|v\|^{2}+\left(\alpha+\delta^{2}-\beta \delta\right)\|u\|^{2}+\|\nabla u\|^{2} .
$$

Same as the second inequality in (46), from the weak convergence shown by (45), for any sequence $\left\{g_{0, m}=\right.$ $\left.\left(u_{0, m}, v_{0, m}\right)\right\}_{m=1}^{\infty} \subset B$, we have

$$
\liminf _{m \rightarrow \infty}\left\|\Phi\left(t_{m}, g_{0, m}\right)\right\|_{\Pi} \geq\|(\tilde{u}, \tilde{v})\|_{\Pi} .
$$

Meanwhile, (76) implies that

$$
\limsup _{m \rightarrow \infty}\left\|\Phi\left(t_{m}, g_{0, m}\right)\right\|_{\Pi} \leq\|(\tilde{u}, \tilde{v})\|_{\Pi} .
$$

Thus we have proved

$$
\lim _{m \rightarrow \infty}\left\|\Phi\left(t_{m}, g_{0, m}\right)\right\|_{\Pi}=\|(\tilde{u}, \tilde{v})\|_{\Pi} .
$$


Finally, for the Hilbert space $H^{1}\left(\mathbb{R}^{n}\right) \times L^{2}\left(\mathbb{R}^{n}\right)$, the weak convergence (45) and the norm convergence (77) imply the strong convergence. Therefore, up to finite steps of subsequence selections (always relabeled as the same), we reach the conclusion that

$$
\lim _{m \rightarrow \infty} \Phi\left(t_{m}, g_{0, m}\right)=(\tilde{u}, \tilde{v}) \quad \text { strongly in } H^{1}\left(\mathbb{R}^{n}\right) \times L^{2}\left(\mathbb{R}^{n}\right) .
$$

Thus the proof is completed.

\section{The Existence of Random Attractor}

In this section we shall first prove an instrumental convergence theorem in the space $L^{p}(X, \mathscr{M}, \mu)$ of Vitali type. It will pave the way to prove asymptotic compactness of the first component of the semiflow $\Phi$ in the space $L^{p}\left(\mathbb{R}^{n}\right)$ for any exponent $1 \leq p \leq \frac{n+2}{n-2}$. This is the crucial and final step to accomplish the proof of the existence of a global attractor for this dynamical system $\Phi$ for the nonlinear damped wave equation (1).

Theorem 8. Let $(X, \mathscr{M}, \mu)$ be a $\sigma$-finite measure space and assume that a sequence $\left\{f_{m}\right\}_{m=1}^{\infty} \subset L^{p}(X, \mathscr{M}, \mu)$ with $1 \leq p<\infty$ satisfies

$$
\lim _{m \rightarrow \infty} f_{m}(x)=f(x) \text {, a.e. }
$$

Then $f \in L^{p}(X, \mathscr{M}, \mu)$ and

$$
\lim _{m \rightarrow \infty}\left\|f_{m}-f\right\|_{L^{p}(X, \mathscr{M}, \mu)}=0
$$

if and only if the following two conditions are satisfied:

(a) For any given $\varepsilon>0$, there exists a set $A_{\varepsilon} \in \mathscr{M}$ such that $\mu\left(A_{\varepsilon}\right)<\infty$ and

$$
\int_{X \backslash A_{\varepsilon}}\left|f_{m}(x)\right|^{p} d \mu<\varepsilon, \quad \text { for all } m \geq 1 .
$$

(b) The absolutely continuous property of the $L^{p}$ integrals is satisfied uniformly, i.e.

$$
\lim _{\mu(Y) \rightarrow 0} \int_{Y}\left|f_{m}(x)\right|^{p} d \mu=0, \quad \text { uniformly for all } m \geq 1 .
$$

Proof. First we prove the necessity.

Statement (a): Under the condition (79), for an arbitrarily given $\varepsilon>0$ there exists an integer $N=N(\varepsilon) \geq 1$ such that

$$
\left\|f_{m}-f\right\|_{L^{p}(X, \mathscr{M}, \mu)}^{p}<\frac{\varepsilon}{2^{p}}, \text { for all } m>N .
$$

Since $f \in L^{p}(X, \mathscr{M}, \mu)$, there exist measurable sets $B_{\varepsilon}$ and $S_{\varepsilon}$ both of finite measure, such that

$$
\int_{X \backslash B_{\varepsilon}}|f(x)|^{p} d \mu<\frac{\varepsilon}{2^{p}} \quad \text { and } \quad \int_{X \backslash S_{\varepsilon}}\left|f_{m}(x)\right|^{p} d \mu<\varepsilon \text {, for } m=1, \cdots, N \text {. }
$$

Put $A_{\varepsilon}=B_{\varepsilon} \cup S_{\varepsilon}$. Then $\mu\left(A_{\varepsilon}\right)<\infty$ and we have

$$
\begin{aligned}
& \int_{X \backslash A_{\varepsilon}}\left|f_{m}(x)\right|^{p} d \mu=\int_{X \backslash A_{\varepsilon}}\left(\left|f_{m}(x)-f(x)\right|+|f(x)|\right)^{p} d \mu \\
\leq & 2^{p-1}\left(\int_{X}\left|f_{m}(x)-f(x)\right|^{p} d \mu+\int_{X \backslash B_{\varepsilon}}|f(x)|^{p} d \mu\right)<\frac{\varepsilon}{2}+\frac{\varepsilon}{2}=\varepsilon \text {, for } m>N .
\end{aligned}
$$

Besides, from the second inequality in (83) it follows that

$$
\int_{X \backslash A_{\varepsilon}}\left|f_{m}(x)\right|^{p} d \mu \leq \int_{X \backslash S_{\varepsilon}}\left|f_{m}(x)\right|^{p} d \mu<\varepsilon, \quad \text { for } m=1, \cdots, N .
$$


Therefore, the statement (a) is valid.

Statement (b): By the absolutely continuous property of Lebesgue integral on a $\sigma$-finite measure space, for any given $\varepsilon>0$, there exists $\delta_{0}=\delta_{0}(\varepsilon)>0$ such that whenever $\mu(Y)<\delta_{0}$ one has

$$
\int_{Y}|f(x)|^{p} d \mu<\frac{\varepsilon}{2^{p}} \quad \text { and } \quad \int_{Y}\left|f_{m}(x)\right|^{p} d \mu<\varepsilon, \quad \text { for } m=1, \cdots, N,
$$

where $N=N(\varepsilon)$ is the same integer in (82). Then for any measurable set $Y \subset X$ with $\mu(Y)<\delta_{0}$ one also has

$$
\int_{Y}\left|f_{m}(x)\right|^{p} d \mu \leq 2^{p-1}\left(\int_{X}\left|f_{m}(x)-f(x)\right|^{p} d \mu+\int_{Y}|f(x)|^{p} d \mu\right)<\varepsilon, \quad \text { for } m>N .
$$

Thus the statement (b) is valid.

Next we prove the sufficiency. Suppose the two conditions (a) and (b) are satisfied. First of all, by the condition (a) and Fatou's Lemma, for an arbitrarily given $\varepsilon>0$ there exists a set $A_{\varepsilon}$ of finite measure with

$$
\sup _{m \geq 1} \int_{X \backslash A_{\varepsilon}}\left|f_{m}(x)\right|^{p} d \mu<\varepsilon,
$$

which implies that the limit function $f$ in the assumption (78) satisfies

$$
\int_{X \backslash A_{\varepsilon}}|f(x)|^{p} d \mu \leq \liminf _{m \rightarrow \infty} \int_{X \backslash A_{\varepsilon}}\left|f_{m}(x)\right|^{p} d \mu<\varepsilon .
$$

Hence it follows that

$$
f \in L^{p}\left(X \backslash A_{\varepsilon}\right) \quad \text { and } \quad\left\|f_{m}-f\right\|_{L^{p}\left(X \backslash A_{\varepsilon}\right)}<2 \varepsilon^{1 / p} \text {, for all } m \geq 1 .
$$

Therefore, the proof of $f \in L^{p}(X, \mathscr{M}, \mu)$ and (79) is reduced to proving that

$$
f \in L^{p}(Y) \text { and } \lim _{m \rightarrow \infty}\left\|f_{m}-f\right\|_{L^{p}(Y)}=0,
$$

for any given measurable set $Y \subset X$ with $\mu(Y)<\infty$.

By the condition (b), for any given $\varepsilon>0$, there exists $\delta_{1}=\delta_{1}(\varepsilon)>0$ such that for any $S \subset X$ with $\mu(S)<\delta_{1}$ one has

$$
\int_{S}\left|f_{m}(x)\right|^{p} d \mu<\varepsilon^{p}, \text { uniformly in } m \geq 1 .
$$

Consequently, by Fatou's lemma,

$$
\int_{S}|f(x)|^{p} d \mu \leq \liminf _{m \rightarrow \infty} \int_{S}\left|f_{m}(x)\right|^{p} d \mu<\varepsilon^{p} .
$$

By Egorov's theorem on Lebesgue integral over such a set $Y$ of finite measure in the space $(X, \mathscr{M}, \mu)$, there exists a measurable subset $B \subset Y$ with $\mu(Y \backslash B)<\delta_{1}$ such that

$$
\lim _{m \rightarrow \infty} f_{m}(x)=f(x), \text { uniformly a.e. on } B,
$$

so that there exists an integer $m_{0}=m_{0}(\varepsilon) \geq 1$ such that

$$
\left\|f_{m}-f\right\|_{L^{p}(B)}<\varepsilon, \text { for all } m>m_{0} .
$$

Combining (88), (89) and (90), we get

$$
\left\|f_{m}-f\right\|_{L^{p}(Y)} \leq\left\|f_{m}\right\|_{L^{p}(Y \backslash B)}+\|f\|_{L^{p}(Y \backslash B)}+\left\|f_{m}-f\right\|_{L^{p}(B)}<3 \varepsilon \text {, for } m>m_{0} .
$$

Therefore, (87) is proved. The proof is completed.

Finally we present and prove the main result of this work on the existence of a global attractor for this semiflow $\Phi$ generated by the nonlinear damped wave equation (1) on the product Banach space with critical exponent and arbitrary space dimension. 
Theorem 9. Under the Standing Assumption, the semiflow $\Phi$ generated by the nonlinear damped wave equation (1) in the converted problem (4) on the space $E=\left(H^{1}\left(\mathbb{R}^{n}\right) \cap L^{p}\left(\mathbb{R}^{n}\right)\right) \times L^{2}\left(\mathbb{R}^{n}\right)$ has a global attractor $\mathscr{A}$ in $E$.

Proof. Lemma 3 shows that there exists an absorbing set, the $K=B_{E}(0, R)$ in the space $E$ for the semiflow $\Phi$. It suffices to prove that the semiflow $\Phi$ is asymptotically compact in $E$.

(1) Theorem 7 shows that for any given bounded set $B \subset E$ and any sequences $t_{m} \rightarrow \infty$ and $\left\{g_{0, m}=\right.$ $\left.\left(u_{0, m}, v_{0, m}\right)\right\} \subset B$, the sequence $\left\{\Phi\left(t_{m}, g_{0, m}\right)\right\}_{m=1}^{\infty}$ has a convergent subsequence, which is relabeled by the same, such that

$$
\Phi\left(t_{m}, g_{0, m}\right) \longrightarrow(\tilde{u}, \tilde{v}) \text { strongly in } H^{1}\left(\mathbb{R}^{n}\right) \times L^{2}\left(\mathbb{R}^{n}\right),
$$

and consequently

$$
\mathbb{P}_{u} \Phi\left(t_{m}, g_{0, m}\right) \longrightarrow \tilde{u} \text { strongly in } L^{2}\left(\mathbb{R}^{n}\right) .
$$

Here $\mathbb{P}_{u}:(u, v) \mapsto u$ is the component projection.

(2) Applying the first item in Lemma 5 to the space $L^{2}\left(\mathbb{R}^{n}\right)$, it follows from (91) that there exists a subsequence $\left\{\Phi\left(t_{m_{k}}, g_{0, m_{k}}\right)\right\}_{k=1}^{\infty}$ of $\left\{\Phi\left(t_{m}, g_{0, m}\right)\right\}_{m=1}^{\infty}$ such that

$$
\lim _{k \rightarrow \infty} \Phi\left(t_{m_{k}}, g_{0, m_{k}}\right)(x)=(\tilde{u}(x), \tilde{v}(x)) \text {, a.e. in } \mathbb{R}^{n} .
$$

Hence we have

$$
\lim _{k \rightarrow \infty} \mathbb{P}_{u} \Phi\left(t_{m_{k}}, g_{0, m_{k}}\right)(x)=\tilde{u}(x), \text { a.e. in } \mathbb{R}^{n} .
$$

Therefore, the assumption (78) in Theorem 8 is satisfied by the sequence of functions $\left\{\mathbb{P}_{u} \Phi\left(t_{m_{k}}, g_{0, m_{k}}\right)(x)\right\}_{k=1}^{\infty}$ in $L^{p}\left(\mathbb{R}^{n}\right)$.

(3) By Lemma 4, for any $\varepsilon>0$, there exists an integer $k_{0}=k_{0}(B, \varepsilon)>0$ and $V=V(\varepsilon) \geq 1$ such that for all $k>k_{0}$ one has

$$
\int_{\mathbb{R}^{n} \backslash B_{V}}\left|\mathbb{P}_{u} \Phi\left(t_{m_{k}}, g_{0, m_{k}}\right)(x)\right|^{p} d x \leq\left\|\Phi\left(t_{m_{k}}, g_{0, m_{k}}\right)\right\|_{E\left(\mathbb{R}^{n} \backslash B_{V}\right)}^{p}<\varepsilon
$$

for any $g_{0, m_{k}} \in B$, where $B_{V}$ is the ball centered at the origin with radius $V$ in $\mathbb{R}^{n}$. Then there exists $V_{0}=V_{0}(\varepsilon)>0$ such that

$$
\int_{\mathbb{R}^{n} \backslash B_{V_{0}}}\left|\mathbb{P}_{u} \Phi\left(t_{m_{k}}, g_{0, m_{k}}\right)(x)\right|^{p} d x<\varepsilon, \quad \text { for } k=1, \cdots, k_{0} .
$$

Thus (95) and (96) confirm that with $A_{\varepsilon}=B_{\max \left\{V, V_{0}\right\}}$ the condition (a) in Theorem 8 is satisfied by the sequence of functions $\left\{\mathbb{P}_{u} \Phi\left(t_{m_{k}}, g_{0, m_{k}}\right)(x)\right\}_{k=1}^{\infty}$ in $L^{p}\left(\mathbb{R}^{n}\right)$.

(4) Finally we prove that the uniform absolutely continuous condition (b) of Theorem 8 is also satisfied by the sequence of functions $\left\{\mathbb{P}_{u} \Phi\left(t_{m_{k}}, g_{0, m_{k}}\right)(x)\right\}_{k=1}^{\infty}$ in $L^{p}\left(\mathbb{R}^{n}\right)$.

According to the Standing Assumption, for any measurable set $Y \subset \mathbb{R}^{n}$, we have

$$
C_{3} \int_{Y}|u|^{p} d x \leq \int_{Y}\left(F(x, u)+\phi_{3}(x)\right) d x \leq \Gamma_{Y}(u, v), \quad \text { for }(u, v) \in E,
$$

where $\Gamma_{Y}(u, v)$ is analogous to (40) and defined by

$$
\Gamma_{Y}(u, v)=\|v\|_{L^{2}(Y)}^{2}+\left(\alpha+\delta^{2}-\beta \delta\right)\|u\|_{L^{2}(Y)}^{2}+\|\nabla u\|_{L^{2}(Y)}^{2}+2 \int_{Y}\left(F(x, u)+\phi_{3}(x)\right) d x .
$$

We can integrate the inequality (14) over the time interval $\left[0, t_{m}\right]$ to get

$$
\begin{aligned}
& \Gamma_{Y}\left(u\left(t_{m}, u_{0, m}\right), v\left(t_{m}, v_{0, m}\right)\right) \\
\leq & e^{-\sigma t_{m}} \Gamma_{Y}\left(u_{0, m}, v_{0, m}\right)+\int_{0}^{t_{m}} e^{-\sigma t}\left(2 \delta\left(C_{2}\left\|\phi_{3}\right\|_{L^{1}}+\left\|\phi_{2}\right\|_{L^{1}}\right)+\frac{\|g\|_{L^{2}(Y)}^{2}}{\beta-\delta}\right) d t .
\end{aligned}
$$


Substitute the expression of $\Gamma_{Y}\left(u_{0, m}, v_{0, m}\right)$ with $\left(u_{0, m}, v_{0, m}\right) \in B$ into the inequality (98). Since (5)-(6) yield

$$
\int_{Y}\left(F(x, u)+\phi_{3}(x)\right) d x \leq \frac{1}{C_{2}}\left[C_{1}\|u\|_{L^{p}(Y)}^{p}+\|u\|_{L^{2}(Y)}^{2}+\left\|\phi_{1}\right\|_{L^{2}(Y)}^{2}+\left\|\phi_{2}\right\|_{L^{1}(Y)}\right],
$$

for any $g_{0, m}=\left(u_{0, m}, v_{0, m}\right) \in B$, we get

$$
\begin{aligned}
& C_{3} \int_{Y}\left|u\left(t_{m}, u_{0, m}\right)\right|^{p} d x \leq \Gamma_{Y}\left(u\left(t_{m}, u_{0, m}\right), v\left(t_{m}, v_{0, m}\right)\right) \\
\leq & e^{-\sigma t_{m}}\left[\left\|v_{0, m}\right\|_{L^{2}(Y)}^{2}+\left(\alpha+\delta^{2}-\beta \delta\right)\left\|u_{0, m}\right\|_{L^{2}(Y)}^{2}+\left\|\nabla u_{0, m}\right\|_{L^{2}(Y)}^{2}\right] \\
& +e^{-\sigma t_{m}} \frac{1}{C_{2}}\left[C_{1}\left\|u_{0, m}\right\|_{L^{p}(Y)}^{p}+\left\|u_{0, m}\right\|_{L^{2}(Y)}^{2}+\left\|\phi_{1}\right\|_{L^{2}(Y)}^{2}+\left\|\phi_{2}\right\|_{L^{1}(Y)}\right] \\
& +2 \delta C_{2} \int_{0}^{t_{m}} e^{-\sigma t}\left(\left\|\phi_{1}\right\|_{L^{2}(Y)}^{2}+\left\|\phi_{3}\right\|_{L^{1}(Y)}\right) d t+\int_{0}^{t_{m}} \frac{e^{-\sigma t}}{\beta-\delta}\|g\|_{L^{2}(Y)}^{2} d t .
\end{aligned}
$$

Due to the absolute continuity of the respective Lebesgue integrals of the functions $\phi_{1}(x), \phi_{2}(x), \phi_{3}(x)$ and $g$ involved in the above inequality (99), for an arbitrarily given $\eta>0$, there exists $\mu_{0}=\mu_{0}(\eta)>0$ such that for any measurable set $Y \subset \mathbb{R}^{n}$ with $\mu(Y)<\mu_{0}$ one has

$$
\begin{aligned}
e^{-\sigma t_{m}} & \frac{1}{C_{2}}\left(\left\|\phi_{1}\right\|_{L^{2}(Y)}^{2}+\left\|\phi_{2}\right\|_{L^{1}(Y)}\right)+2 \delta C_{2} \int_{0}^{t_{m}} e^{-\sigma t}\left(\left\|\phi_{1}\right\|_{L^{2}(Y)}^{2}+\left\|\phi_{3}\right\|_{L^{1}(Y)}\right) d t+\int_{0}^{t_{m}} \frac{e^{-\sigma t}}{\beta-\delta}\|g\|_{L^{2}(Y)}^{2} d t \\
& \leq \frac{1}{C_{2}}\left(\left\|\phi_{1}\right\|_{L^{2}(Y)}^{2}+\left\|\phi_{2}\right\|_{L^{1}(Y)}\right)+\frac{2 \delta}{\sigma} C_{2}\left(\left\|\phi_{1}\right\|_{L^{2}(Y)}^{2}+\left\|\phi_{3}\right\|_{L^{1}(Y)}\right)+\frac{1}{\sigma(\beta-\delta)}\|g\|_{L^{2}(Y)}^{2}<\frac{\eta}{2} .
\end{aligned}
$$

Moreover, since $B \subset E$ is a bounded set, there exists a constant $\hat{C}>0$ such that

$$
\begin{gathered}
e^{-\sigma t_{m}}\left[\left\|v_{0, m}\right\|_{L^{2}(Y)}^{2}+\left(\alpha+\delta^{2}-\beta \delta\right)\left\|u_{0, m}\right\|_{L^{2}(Y)}^{2}+\left\|\nabla u_{0, m}\right\|_{L^{2}(Y)}^{2}\right] \\
+\frac{1}{C_{2}} e^{-\sigma t_{m}}\left[C_{1}\left\|u_{0, m}\right\|_{L^{p}(Y)}^{p}+\left\|u_{0, m}\right\|_{L^{2}(Y)}^{2}\right] \leq e^{-\sigma t_{m}} \hat{C}\left(\|B\|_{E(Y)}^{2}+\|B\|_{E(Y)}^{p}\right),
\end{gathered}
$$

where $\|B\|_{E(Y)}=\max _{g_{0} \in B\left(\theta_{-t_{m}} \omega\right)}\left\|g_{0} \zeta_{Y}\right\|_{E}$ with $\zeta_{Y}$ being the characteristic function for the set $Y$. Clearly,

$$
\lim _{t \rightarrow \infty} e^{-\sigma t}\|B\|_{E}=0
$$

For the aforementioned arbitrary $\eta>0$, there exists an integer $m_{0}=m_{0}(B, \eta) \geq 1$ such that

$$
e^{-\sigma t_{m}} \hat{C}\left(\|B\|_{E(Y)}^{2}+\|B\|_{E(Y)}^{p}\right) \leq e^{-\sigma t_{m}} \hat{C}\left(\|B\|_{E}^{2}+\|B\|_{E}^{p}\right)<\frac{\eta}{2}
$$

for all $m>m_{0}$. Then there esists $\mu_{1}=\mu_{1}\left(B, m_{0}, \eta\right)>0$ such that for any set $Y$ with $\mu(Y)<\mu_{1}$ one has

$$
e^{-\sigma t_{j}} \hat{C}\left(\|B\|_{E(Y)}^{2}+\|B\|_{E(Y)}^{p}\right)<\frac{\eta}{2}, \quad j=1, \cdots, m_{0} .
$$

Put together (100), (101) and (102) with (99). It shows that

$$
C_{3} \int_{Y}\left|u\left(t_{m}, u_{0, m}\right)\right|^{p} d x \leq \frac{\eta}{2}+\frac{\eta}{2}=\eta, \text { for all } m \geq 1,
$$

whenever a measurable set $Y \subset \mathbb{R}^{n}$ satisfies $\mu(Y)<\min \left\{\mu_{0}, \mu_{1}\right\}$. Therefore,

$$
\lim _{\mu(Y) \rightarrow 0} \int_{Y}\left|\mathbb{P}_{u} \Phi\left(t_{m_{k}}, g_{0, m}\right)(x)\right|^{p} d x=0 \text { uniformly for all } k \geq 1,
$$


so that the condition (b) of Theorem 8 is also satisfied by the sequence of functions $\left\{\mathbb{P}_{u} \Phi\left(t_{m_{k}}, g_{0, m_{k}}\right)(x)\right\}_{k=1}^{\infty}$ in $L^{p}\left(\mathbb{R}^{n}\right)$.

As checked by the above steps (2), (3) and (4) in this proof, all the conditions in Theorem 8 are satisfied by the sequence of functions $\left\{\mathbb{P}_{u} \Phi\left(t_{m_{k}}, g_{0, m_{k}}\right)(x)\right\}_{k=1}^{\infty}$ in $L^{p}\left(\mathbb{R}^{n}\right)$. Then we apply Theorem 8 to obtain

$$
\lim _{k \rightarrow \infty} \mathbb{P}_{u} \Phi\left(t_{m_{k}}, g_{0, m_{k}}\right)=\tilde{u} \text { strongly in } L^{p}\left(\mathbb{R}^{n}\right) .
$$

Finally, combination of (91) and (105) shows that there exists a convergent subsequence $\left\{\Phi\left(t_{m_{k}}, g_{0, m_{k}}\right)\right\}_{k=1}^{\infty}$ of the sequence $\left\{\Phi\left(t_{m}, g_{0, m}\right)\right\}_{m=1}^{\infty}$ in the space $E=\left(H^{1}\left(\mathbb{R}^{n}\right) \cap L^{p}\left(\mathbb{R}^{n}\right)\right) \times L^{2}\left(\mathbb{R}^{n}\right)$. Therefore, the semiflow $\Phi$ on the Banach space $E$ is asymptotically compact.

According to Theorem 1, we conclude that there exists a global attractor $\mathscr{A}$ in $E$ for this semiflow $\Phi$ generated by the original nonlinear damped wave equation (1). The proof is completed.

\section{References}

[1] J. M. Arrieta, A. N. Carvalho and J. K. Hale. (1992), A damped hyperbolic equation with critical exponent, Communications in Partial Differential Equations, 17, 841-866. doi 10.1080/036053092088020866

[2] A. V. Babin and M. I. Vishik. (1992), Attractors of Evolution Equations, North-Holland, Amsterdam.

[3] J. M. Ball. (2004), Global attractors for damped semilinear wave equations, Discrete and Continuous Dynamical Systems, 10, 31-52. doi 10.3934/dcds.2004.10.31

[4] V. Belleri and V. Pata. (2001), Attractors for semilinear strongly damped wave equations on $\mathbb{R}^{3}$, Discrete and Continuous Dynamical Systems, Ser. A, 7, 719-735. doi 10.3934/dcds.2001.7.719

[5] H. Brezis. (2011), Functional Analysis, Sobolev Spaces and Partial Differential Equations, Springer-Verlag, New York.

[6] A.N. Carvalho and J.W. Cholewa. (2002), Attrarctors for strongly damped wave equations with critical nonlinearities, Pacific Journal of Mathematics. 207, 287-310. doi 10.2140/pjm.2002.207.287

[7] T. Caraballo, A. N. Carvalho, J. A. Langa and F. Rivero. (2011), A non-autonomous strongly damped wave equation: Existence and continuity of the pullback attractor, Nonlinear Analysis: Theory, Methods and Applications, 74, 22722283. doi 10.1016/j.na.2010.11.032

[8] V. V. Chepyzhov and M. I. Vishik. (2002), Attractors for Equations of Mathematical Physics, American Mathematical Society, Providence, R.I.

[9] H. Crauel, A. Debussche and F. Flandoli. (1997), Random attractors, Journal of Dynamics and Differential Equations, 9, 307-341. doi 10.1007/BF02219225

[10] J. Duan, K. Lu and B. Schmalfuss. (2003), Invariant manifolds for stochastic partial differential equations, The Annals of Probability, 31, 2109-2135.

[11] X. Fan. (2008), Random attractor for a damped stochastic wave equation with multiplicative noise, International Journal of Mathematics, 19, 421-437. doi 10.1142/S0129167X08004741

[12] E. Feireisl. (1994), Attractors for semilinear damped wave equations on $\mathbb{R}^{3}$, Nonlinear Analysis: Theory, Methods and Applications, 23, 187-195. doi 10.1016/0362546X(94)900418

[13] E. Feireisl. (1995), Global attractors for damped wave equations with supercritical exponent, J. Differential Equations, 116, 431-447. doi 10.1006/jdeq.1995.1042

[14] J. M. Ghidaglia and R. Temam. (1987), Attractors of damped nonlinear hyperbolic equations, J. Math. Pures Appl., 66, 273-319.

[15] R. Jones and B. Wang. (2013), Asymptotic behavior of a class of stochastic nonlinear wave equations with dispersive and dissipative terms, Nonlinear Analysis: Real World Applications, 14, 1308-1322. doi 10.1016/j.nonrwa.2012.09.019

[16] A. Kh. Khanmamedov. (2006), Global attractors for wave equations with nonlinear interior damping and critical exponents, Journal of Differential Equations, 230, 702-719. doi 10.1016/j.jde.2006.06.001

[17] H. Li and Y. You. (2015), Random attractor for stochastic wave equation with arbitrary exponent and additive noise on $\mathbb{R}^{n}$, Dynamics of Partial Differential Equations, 12, 343-378. doi 10.4310/DPDE.2015.v12.n4.a3

[18] H. Li, Y. You and J. Tu. (2015), Random attractors and averaging for nonautonomous stochastic wave equations with nonlinear damping, Journal of Differential Equations, 258, 148-190. doi 10.1016/j.jde.2014.09.007

[19] M. Nakao. (2006), Global attractors for nonlinear wave equations with nonlinear dissipative terms, J. Differential Equations, 227, 204-229. doi 10.1016/j.jde.2005.09.013

[20] R. Sell and Y. You. (2002), Dynamics of Evolutionary Equations, Springer-Verlag, New York.

[21] C. Sun, M. Yang and C. Zhong. (2006), Global attractors for the wave equation with nonlinear damping, Journal of Differential Equations, 227, 427-443. doi 10.1016/j.jde.2005.09.010

[22] R. Temam. (1997), Infinite-Dimensional Dynamical Systems in Mechanics and Physics, Springer-Verlag, New York. 
[23] B. Wang. (2011), Asymptotic behavior of stochastic wave equations with critical exponents on $R^{3}$, Transactions of the American Mathematical Society, 363, 3639-3663. doi 10.1090/S0002-9947-2011-05247-5

[24] B. Wang. (2014), Random attractors for non-autonomous stochastic wave equations with multiplicative noise, Discrete and Continuous Dynamical Systems, 34, 269-300. doi 10.3934/dcds.2014.34.269

[25] M. Yang, J. Duan and P. Kloeden. (2011), Asymptotic behavior of solutions for random wave equation with nonlinear damping and white noise, Nonlinear Analysis: Real World Applications, 12, 464-478. doi 10.1016/j.nonrwa.2010.06.032

[26] Y. You. (2004), Global dynamics of nonlinear wave equations with cubic non-monotone damping, Dynamics of Partial Differential Equations, 1, 65-86. doi 10.4310/DPDE.2004.v1.n1.a3

(OUP4 Sciences. All rights reserved. 